\title{
KP hierarchy for the cyclic quiver
}

Cite as: J. Math. Phys. 58, 071702 (2017); https://doi.org/10.1063/1.4991031

Submitted: 31 March 2017 . Accepted: 18 June 2017 . Published Online: 12 July 2017

Oleg Chalykh, and Alexey Silantyev

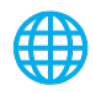

View Online
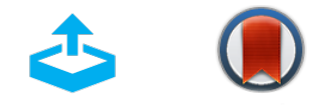

\section{ARTICLES YOU MAY BE INTERESTED IN}

Fock representations of Q-deformed commutation relations

Journal of Mathematical Physics 58, 073501 (2017); https://

doi.org/10.1063/1.4991671

Particle-like structure of Lie algebras

Journal of Mathematical Physics 58, 071703 (2017); https://

doi.org/10.1063/1.4991657

The second bosonization of the CKP hierarchy

Journal of Mathematical Physics 58, 071707 (2017); https://

doi.org/10.1063/1.4990795

\section{Where in the world is AIP Publishing?}

Find out where we are exhibiting next 


\title{
KP hierarchy for the cyclic quiver
}

\author{
Oleg Chalykh ${ }^{\mathrm{a})}$ and Alexey Silantyev ${ }^{\mathrm{b})}$ \\ School of Mathematics, University of Leeds, Leeds LS2 9JT, United Kingdom
}

(Received 31 March 2017; accepted 18 June 2017; published online 12 July 2017)

\begin{abstract}
We introduce a generalisation of the KP hierarchy, closely related to the cyclic quiver and the Cherednik algebra $H_{k}\left(\mathbb{Z}_{m}\right)$. This hierarchy depends on $m$ parameters (one of which can be eliminated), with the usual KP hierarchy corresponding to the $m=1$ case. Generalising the result of Wilson [Invent. Math. 133(1), 1-41 (1998)], we show that our hierarchy admits solutions parameterised by suitable quiver varieties. The pole dynamics for these solutions is shown to be governed by the classical CalogeroMoser system for the wreath-product $\mathbb{Z}_{m} \succ S_{n}$ and its new spin version. These results are further extended to the case of the multi-component hierarchy. Published by AIP Publishing. [http://dx.doi.org/10.1063/1.4991031]
\end{abstract}

\section{INTRODUCTION}

It is a remarkable fact, first observed in Ref. 1, that integrable non-linear PDEs admit solutions whose poles move as classical-mechanical particles in a completely integrable fashion. One of the best-known examples is the KP hierarchy, in which case the classical integrable system in question is the Calogero-Moser system, see Refs. 37 and 15. Since the Calogero-Moser system can be generalised to all root systems and Coxeter groups, ${ }^{44,45}$ there is a natural question whether these generalisations can be similarly linked to integrable non-linear equations. For the infinite series of classical root systems, a partial answer can be obtained by folding, ${ }^{10,43}$ which relates the Calogero-Moser systems in types $A$ and $B$. However, what can be obtained in type $B$ by folding constitutes only a very special case, while for the most general coupling parameters, the answer, to the best of our knowledge, remained unknown. The present paper gives an answer to this question for all classical root systems and much more: as we will explain, there is a natural generalisation of the KP hierarchy that admits rational solutions whose pole dynamics is governed by the Calogero-Moser system for the complex reflection group $\mathbb{Z}_{m} \prec S_{n}$. The case $m=1$ is the usual, type $A$ Calogero-Moser problem, and the case $m=2$ covers the remaining classical types.

Our approach to this problem is inspired by a result of G. Wilson, who has shown in Ref. 51 that rational solutions of the KP hierarchy can be parametrised by (the points of) certain affine varieties, the Calogero-Moser spaces. The $n$th Calogero-Moser space $\mathcal{C}_{n}$ can be viewed as a completed phase space of the $n$-particle Calogero-Moser system (hence their name). On the other hand, $\mathcal{C}_{n}$ is a particular example of a quiver variety, with the underlying quiver consisting of just one loop. From that perspective, the present work is a generalisation of that of Wilson, with the one-loop quiver replaced by the cyclic quiver on $m$ vertices. It is worth mentioning that the spaces $\mathcal{C}_{n}$ have also appeared in the context of non-commutative projective geometry and classification of ideals of the first Weyl algebra. ${ }^{7,8,36}$ The results of Ref. 7 prompted Crawley-Boevey and Holland to conjecture a similar link in the case of affine Dynkin quivers (see Ref. 17, p. 45). Their conjecture was later generalised and proved by Baranovsky, Ginzburg, and Kuznetsov in Refs. 4 and 5. In the case of the

\footnotetext{
${ }^{a)}$ E-mail: o.chalykh@leeds.ac.uk

${ }^{b}$ Current address: Bogoliubov Laboratory of Theoretical Physics, JINR, 141980 Dubna, Moscow Region, Russia and Dubna State University, 19 Universitetskaya St., 141980 Dubna, Moscow Region, Russia. Electronic mail: aleksejsilantjev@gmail.com.
} 
cyclic quiver, this is related to the problem of classifying ideals of the Cherednik spherical subalgebra $\mathbf{e} H_{k} \mathbf{e}$ for the cyclic group. In Ref. 26, this problem was tackled differently to Refs. 4 and 5 by using the approach of Ref. 11. In fact, some of our formulas and calculations closely resemble those in Refs. 11 and 26.

While the moduli spaces parametrising solutions to our hierarchy are known from the above works, the hierarchy itself seems new. We construct it in a Lax form in the same spirit as the usual KP hierarchy, but replacing the operator $\partial=d / d x$ with the one-variable Dunkl operator for $\Gamma=\mathbb{Z}_{m}$. Solutions to the hierarchy for given quiver data are described by an explicit formula, so checking that they satisfy the equations of the hierarchy amounts to a direct algebraic calculation. Note that we do not require a passage to an adelic Grassmannian $\mathrm{Gr}^{\text {ad }}$ as in Ref. 51 (for the details of how to define the appropriate notion in the present context, see Refs. 3 and 5).

It is worth mentioning that our initial motivation for the present work was to understand the moduli space of bispectral rings of differential operators constructed by Bakalov, Horozov, and Yakimov in Ref. 3. An attempt in that direction, also inspired by Ref. 51, was made by Rothstein in Ref. 46, but the proofs in loc. cit. were omitted. Moreover, as our work shows, the answer to that question turns out to be simpler and more natural than it might be suggested by Ref. 46. Our results also provide an answer to a question raised by Ref. 31. Namely, in Ref. 31 they study algebraically integrable operators with $\mathbb{Z}_{m}$-symmetry and ask for a nonlinear hierarchy governing these operators (see Ref. 31, Remark 4.8). The answer (in the case of operators with rational coefficients) is given by the hierarchy constructed in the present paper. Apart from brief remarks in the concluding section, however, we do not discuss these two problems in detail here, leaving them to a separate publication.

Our generalisation of the KP hierarchy is naturally related not just to a cyclic quiver but to a suitable framing of it, obtained by adding a vertex and a number of arrows. Different choices of the framing lead to different hierarchies and to different integrable multi-particle systems governing the pole dynamics. In the simplest case of one added arrow, the classical-mechanical system in question can be identified with the rational Calogero-Moser system with the symmetry group $G=\mathbb{Z}_{m} 2 S_{n}$, while the other cases naturally lead to spin versions of that system. In the case $m$ $=1$, one recovers the Gibbons-Hermsen system, ${ }^{32}$ while in general the systems we obtain this way seem new. Note that there exist various versions of the spin Calogero-Moser system associated with simple complex Lie algebras, see Ref. 39 and references therein. However, to the best of our knowledge, spin versions of the Calogero-Moser system for $G=\mathbb{Z}_{m} \prec S_{n}$ and $m>2$ did not appear in the literature previously. Because we define these systems as a commutative family of Hamiltonian flows on suitable Calogero-Moser spaces, the fact that the flows are complete is obvious from the start. As another by-product, we also find a Lax matrix for the (spin) CalogeroMoser system for $G=\mathbb{Z}_{m} \imath S_{n}$. Note that $G$ is not a Coxeter group for $m>2$, so the corresponding Calogero-Moser system and its Lax matrix cannot be obtained within the general framework of Ref. 9.

The plan of the paper is as follows. In Sec. II, we recall the definition of the Cherednik algebra for $\Gamma=\mathbb{Z}_{m}$, use its microlocalisation to construct our version of the KP hierarchy in a Lax form, and introduce its spherical sub-hierarchy. In Sec. III, we recall the definition of deformed preprojective algebras for an arbitrary quiver $Q$ due to Ref. 16 and define quiver varieties associated with a framing of $Q$. We spell this out in detail for a cyclic quiver $Q_{0}$. By analogy with Ref. 51, one calls these varieties as Calogero-Moser spaces. In Sec. IV, we obtain our main results: we introduce a natural Hamiltonian dynamical system on a Calogero-Moser space and construct rational solutions of our hierarchy starting from any point of this space. A similar result is obtained in Sec. IV C for the spherical sub-hierarchy. The integrable systems governing the pole dynamics for the constructed solutions are considered in more detail in Sec. V. For that we first recall a link, due to Etingof and Ginzburg, ${ }^{27}$ between the Calogero-Moser spaces and Cherednik algebras for $G=\mathbb{Z}_{m} \prec S_{n}$. We use this link to identify the flows of the spherical sub-hierarchy with the Calogero-Moser system for the wreath-product $\mathbb{Z}_{m} \prec S_{n}$. The particle system related to the full hierarchy is considered in Sec. V C; it can be viewed as a spin version of the Calogero-Moser system for $\mathbb{Z}_{m} \prec S_{n}$, where the spin variables belong to the group algebra $\mathbb{C Z}_{m}$. Section VI describes the multi-component version of our hierarchy; this extends the results of the previous two sections and leads to a construction of the general spin 
version of the Calogero-Moser system for $G=\mathbb{Z}_{m} \prec S_{n}$. The concluding section discusses some open questions, further directions, and links.

\section{CHEREDNIK ALGEBRA FOR THE CYCLIC GROUP AND GENERALISED KP HIERARCHY}

\section{A. The Cherednik algebra of $\Gamma=\mathbb{Z}_{m}$}

Let $\Gamma=\left\{e, s, \ldots, s^{m-1}\right\}$ be the multiplicative cyclic group of order $m$ with generator $s$. Identifying $\Gamma$ with the group $\mathbb{Z}_{m}$ of roots of unity, we have an action of $\Gamma$ on $\mathbb{C}$, with $s$ acting as the multiplication by a fixed primitive $m$ th root of unity, $\mu$. This induces an action of $\Gamma$ on the ring $\mathbb{C}(x)$ of rational functions and differential operators of one complex variable. Explicitly, $s(f)(x)=f\left(\mu^{-1} x\right)$ for $f \in \mathbb{C}(x)$, and $s(\partial)=\mu \partial, \partial:=\frac{d}{d x}$. Therefore, we can form the crossed product $\mathcal{D}(x, \partial) * \Gamma$, where $\mathcal{D}(x, \partial)$ denotes the ring of differential operators in $x$ with rational coefficients. Recall that for a group $G$ acting on an algebra $A$, the crossed product $A * G$ is spanned by the pairs $(a, g)$ with $a \in A, g \in G$, with the multiplication defined by $(a, g)(b, h)=(a g(b), g h)$. Abbreviating $(a, g)$ as $a g$, the multiplication consists of moving the group elements to the right using the rule $g a=g(a) g$ so that $(a g)(b h)$ $=a g(b) g h$. For instance, in our situation, we have

$$
s x=\mu^{-1} x s \quad \text { and } \quad s \partial=\mu \partial s .
$$

The differential filtration on the ring $\mathcal{D}(x, \partial)$ extends to a filtration on $\mathcal{D}(x, \partial) * \Gamma$, by placing the elements of $\Gamma$ in degree 0 .

The group algebra $\mathbb{C} \Gamma$ has a natural basis given by the idempotents

$$
\epsilon_{i}=\frac{1}{m} \sum_{r=0}^{m-1} \mu^{-i r} s^{r} \in \mathbb{C}[\Gamma] \quad(i=0, \ldots, m-1) .
$$

Given $k=\left(k_{0}, \ldots, k_{m-1}\right) \in \mathbb{C}^{m}$ and $\tau \neq 0$, one defines the Cherednik algebra $H_{\tau, k}(\Gamma)$ as the subalgebra of $\mathcal{D}(x, \partial) * \Gamma$, generated by $s, x$, and the following element, called Dunkl operator:

$$
y=\tau \partial-\frac{1}{x} \sum_{i=0}^{m-1} m k_{i} \epsilon_{i} .
$$

It is clear from (2.1) that $s y=\mu y s$ and that

$$
\epsilon_{i} x=x \epsilon_{i+1}, \quad \epsilon_{i} y=y \epsilon_{i-1} .
$$

(Here and in similar situations below, the indices are always taken modulo $m$.) Also, the following relation between $x$ and $y$ is easy to check:

$$
x y-y x=\sum_{i=0}^{m-1} \lambda_{i} \epsilon_{i}, \quad \text { with } \quad \lambda_{i}=m\left(k_{i-1}-k_{i}\right)-\tau .
$$

It follows that the algebra $H_{\tau, k}$ can be described by generators and relations as

$$
H_{\tau, k} \simeq(\mathbb{C}\langle x, y\rangle * \Gamma) /(x y-y x-\lambda), \quad \lambda=\sum_{i=0}^{m-1} \lambda_{i} \epsilon_{i} \in \mathbb{C} \Gamma,
$$

where $\mathbb{C}\langle x, y\rangle$ denotes the free algebra on $x, y$ and the action of $\Gamma$ is given by $s(x)=\mu^{-1} x$ and $s(y)=\mu y$.

The algebra $H_{\tau, k}$ is a particular example of a rational Cherednik algebra, which can be associated with any complex reflection group (see Ref. 27). It has a Poincaré-Birkhoff-Witt (PBW) property, namely, a vector space isomorphism $H_{\tau, k} \simeq \mathbb{C}[x] \otimes \mathbb{C} \Gamma \otimes \mathbb{C}[y]$, which means that every element can be uniquely presented as a linear combination of the terms $x^{i} \gamma y^{j}$ with $i, j \geqslant 0$ and $\gamma \in \Gamma$. The inclusion $H_{\tau, k} \subset \mathcal{D}(x, \partial) * \Gamma$ induces a filtration on $H_{\tau, k}$, called differential filtration, with $\operatorname{deg} y=1, \operatorname{deg} x=0$, and $\operatorname{deg} \gamma=0$.

Algebra (2.6) has also appeared in the context of non-commutative deformations of Kleinian singularities, ${ }^{16,34}$ and it is isomorphic to the deformed preprojective algebra of a cyclic quiver as 
defined in Ref. 16 (we will recall that definition and isomorphism in Sec. III below). Note that if we rescale $\tau$ and $k_{i}$ simultaneously, the resulting Cherednik algebra will not change. For $\tau=0$, one defines $H_{0, k}$ by replacing the Dunkl embedding with its quasi-classical counterpart, see Ref. 27; this agrees with the isomorphism (2.6).

\section{B. Microlocalisation of $\boldsymbol{H}_{\tau, k}$}

The well-known construction of the KP hierarchy uses the ring of pseudo-differential operators $\mathcal{D}(x, \partial)\left(\left(\partial^{-1}\right)\right)$. Our generalisation is based instead on a microlocalisation $(\mathcal{D}(x, \partial) * \Gamma)\left(\left(y^{-1}\right)\right)$, where $y$ is the Dunkl operator (2.3). To construct such a microlocalisation explicitly, we will first consider the Ore localisation of $H_{\tau, k}$ at $y$ and then pass to a completion.

We begin by introducing the parameters $c_{\gamma}, \gamma \in \Gamma$ determined from

$$
\sum_{i=0}^{m-1} m k_{i} \epsilon_{i}=\sum_{\gamma \in \Gamma} c_{\gamma} \gamma
$$

Explicitly, $c_{\gamma}=\sum_{i=0}^{m-1} \mu^{-i j} k_{i}$ for $\gamma=s^{j}$. The following relation in $H_{\tau, k}$ is immediate from (2.3) and (2.7):

$$
y a-a y=\tau \partial(a)-\sum_{\gamma \in \Gamma \backslash\{e\}} c_{\gamma} x^{-1}(\gamma a-a \gamma) \quad \text { for any } a=a(x) \in \mathbb{C}[x] .
$$

A similar commutation relation holds for any $f \in \mathbb{C}[x] * \Gamma$. Namely, let us define an algebra automorphism $\theta$ of $\mathbb{C}[x] * \Gamma$ by $\theta(s)=\mu s$ and $\theta(x)=x$. In other words,

$$
\theta\left(\sum_{i=0}^{m-1} a_{i}(x) s^{i}\right)=\sum_{i=0}^{m-1} a_{i}(x) \mu^{i} s^{i} .
$$

Clearly, $\theta^{m}=1$. With this notation, one has

$$
y f-\theta^{-1}(f) y=\tau \partial(f)-\sum_{\gamma \in \Gamma \backslash\{e\}} c_{\gamma} x^{-1}(\gamma f-f \gamma) \quad \text { for any } f \in \mathbb{C}[x] * \Gamma .
$$

Here $\partial$ acts on $\mathbb{C}[x] * \Gamma$ by the formula $\partial\left(\sum_{i} a_{i}(x) s^{i}\right)=\sum_{i} \partial a_{i}(x) s^{i}$. Note that for $a=\sum_{i} a_{i}(x) s^{i}$, we have

$$
x^{-1}\left(s^{j} a-a s^{j}\right)=\sum_{i} x^{-1}\left(a_{i}\left(\mu^{-j} x\right)-a_{i}(x)\right) s^{i+j},
$$

therefore, the denominators in (2.8) cancel, as expected. The right-hand side of (2.8) defines a linear $\operatorname{map} D: \mathbb{C}[x] * \Gamma \rightarrow \mathbb{C}[x] * \Gamma$, that is,

$$
D(f)=\tau \partial(f)-\sum_{\gamma \in \Gamma \backslash\{e\}} c_{\gamma} x^{-1}[\gamma, f] .
$$

From (2.8) we have $y f=\theta^{-1}(f) y+D(f)$. By induction, for any $n \in \mathbb{N}$,

$$
y^{n} f=\sum_{p=0}^{n} \sum_{\substack{i_{0}, \ldots, i_{p} \geqslant 0 \\ i_{0}+\cdots+i_{p}=n-p}} \theta^{-i_{0}} D \theta^{-i_{1}} \ldots D \theta^{-i_{p}}(f) y^{n-p} .
$$

Note that an application of $D$ to a polynomial in $\mathbb{C}[x] * \Gamma$ lowers its degree, therefore the right-hand side of (2.10) will terminate at $p=\operatorname{deg}(f)$. This implies that the set $\left\{y^{k} \mid k \in \mathbb{N}\right\}$ is (right and left) Ore, so let $H_{\tau, k}\left[y^{-1}\right]$ be the corresponding right localisation. By the PBW property of $H_{\tau, k}$, every element of $H_{\tau, k}\left[y^{-1}\right]$ has a unique presentation as a finite sum $u=\sum_{i \in \mathbb{Z}} u_{i} y^{i}$ with $u_{i} \in \mathbb{C}[x] * \Gamma$. The explicit form of the multiplication in $H_{\tau, k}\left[y^{-1}\right]$ can be derived from (2.10). Namely, one first finds that

$$
y^{-1} f=\sum_{p=0}^{\infty}(-1)^{p}(\theta D)^{p} \theta(f) y^{-1-p} .
$$


From that, using induction, we get

$$
y^{-n} f=\sum_{p=0}^{\infty}(-1)^{p} \sum_{\substack{\varepsilon_{1}+\cdots+\varepsilon_{n+p-1}=p \\ \varepsilon_{i} \in\{0,1\}}} \theta D^{\varepsilon_{1}} \theta D^{\varepsilon_{2}} \ldots D^{\varepsilon_{n+p-1}} \theta(f) y^{-n-p} .
$$

Note that if $f$ is not polynomial but, say, rational, $f \in \mathbb{C}(x) * \Gamma$, then the sum in (2.11) and (2.12) is no longer finite. Therefore, in analogy with the notion of formal pseudo-differential operators, let us introduce

$$
\mathcal{P}=\left\{\sum_{i \geqslant 0} f_{j} y^{N-j} \mid f_{j} \in \mathbb{C}(x) * \Gamma, N \in \mathbb{Z}\right\} .
$$

Addition in $\mathcal{P}$ is obvious, while the multiplication is determined by rules (2.10)-(2.12). The fact that the multiplication is associative follows from its associativity in $H_{\tau, k}\left[y^{-1}\right]$. Indeed, checking the associativity in every degree in $y$ requires checking an algebraic relation involving a finite number of the coefficients $f_{j} \in \mathbb{C}(x) * \Gamma$ and operations $\theta$ and $D$. Since we know that this relations hold for arbitrary polynomial coefficients $f_{j}$, they must hold identically. Hence, the multiplication in $\mathcal{P}$ is associative; note that it depends on the parameters $\tau, k$ entering into the definition of $D$.

Fixing $N$ in the definition of $\mathcal{P}$, one gets a subspace $\mathcal{P}_{N} \subset \mathcal{P}$ and an increasing chain $\ldots \subset \mathcal{P}_{N}$ $\subset \mathcal{P}_{N+1} \subset \ldots$, which makes $\mathcal{P}$ into a filtered ring. For any $S=\sum_{j \geqslant 0} f_{j} y^{N-j}$ in $\mathcal{P}$, we have $S=S_{-}+S_{-}$ where

$$
S_{-}=\sum_{j>N} f_{j} y^{N-j}, \quad S_{+}=\sum_{j=0}^{N} f_{j} y^{N-j} .
$$

(In the case $N<0$, we put $S_{-}=S$ and $S_{+}=0$.) This defines a direct sum decomposition $\mathcal{P}=\mathcal{P}_{-} \oplus \mathcal{P}_{+}$ with

$$
\mathcal{P}_{-}=\mathcal{P}_{-1}=\left\{\sum_{j \geqslant 1} f_{j} y^{-j}\right\} \quad \text { and } \quad \mathcal{P}_{+}=\left\{\sum_{j=0}^{N} f_{j} y^{j} \mid N \geqslant 0\right\} .
$$

From the formula for $y$, it easily follows that $\mathcal{P}_{+}=\mathcal{D}(x, \partial) * \Gamma$. The following properties of the multiplication in the ring $\mathcal{P}$ should be clear:

$$
(S f)_{ \pm}=S_{ \pm} \cdot f \quad \forall S \in \mathcal{P}, \forall f \in \mathbb{C}(x) * \Gamma .
$$

Remark 2.1. An alternative construction of the ring $\mathcal{P}$ is by applying microlocalisation in the sense of Ref. 2. Indeed, $\mathcal{P}$ is isomorphic to the microlocalisation $\mathcal{Q}_{S}^{\mu}$ of $R=\mathcal{D}(x, \partial) * \Gamma$ at the multiplicatively closed set $S=\left\{y^{k} \mid k \in \mathbb{N}_{0}\right\}$. This can be checked either at the level of the associated graded rings, using Propositions 3.5 and 3.10 of loc. cit., or by direct comparison.

Remark 2.2. In the definition of $\mathcal{P}$, one can set the coefficients $f_{j}$ to be from any ring $\mathcal{F} * \Gamma$, closed under the action of $D$. For instance, we may replace $\mathcal{F}=\mathbb{C}(x)$ by $\mathbb{C}[[x]], \mathbb{C}((x))$, or by the rings of functions, analytic (respectively, meromorphic) near $x=0$. We may further replace any such $\mathcal{F}$ by a ring of matrices $\operatorname{Mat}(n, \mathcal{F})$.

Remark 2.3. Formulas (2.10) and (2.12) can be rewritten using the operations $D_{i}:=\theta^{-i+1} D \theta^{i}$. Then for any $n \in \mathbb{N}$, one has

$$
\begin{aligned}
y^{n} \theta^{n}(f) & =\sum_{\varepsilon_{1}, \ldots, \varepsilon_{n} \in\{0,1\}} D_{1}^{\varepsilon_{1}} \ldots D_{n}^{\varepsilon_{n}}(f) y^{n-\varepsilon_{1}-\cdots-\varepsilon_{n}}, \\
y^{-n} \theta^{-n}(f) & =\sum_{p=0}^{\infty}(-1)^{p} \sum_{\substack{l_{1}, \ldots, l_{n} \geqslant 0 \\
l_{1}+\cdots+l_{n}=p}}\left(D_{0}\right)^{l_{n}}\left(D_{-1}\right)^{l_{n-1}} \ldots\left(D_{1-n}\right)^{l_{1}}(f) y^{-n-p} .
\end{aligned}
$$




\section{Generalised KP hierarchy}

Let $\mathcal{P}$ be as in Remark 2.2 above, i.e., we allow arbitrary coefficients $f_{j} \in \mathcal{F} * \Gamma$, where $\mathcal{F}$ is either the ring $\mathbb{C}(x)$ of rational functions or one of its variants. Consider an element $L \in \mathcal{P}$ of the form

$$
L=y+\sum_{i \geqslant 0} f_{i} y^{-i},
$$

where the coefficients $f_{i} \in \mathcal{F} * \Gamma$ depend on an infinite number of additional variables $t_{1}, t_{2}, \ldots$, called times. Our generalisation of the KP hierarchy is the following system of equations:

$$
\frac{\partial L}{\partial t_{k}}=\left[\left(L^{k}\right)_{+}, L\right], \quad k=1,2, \ldots
$$

Here $\left(L^{k}\right)_{+}$denotes the positive part of $L^{k}$ according to notation $(2.14)$, and $[\cdot, \cdot]$ is the commutator bracket, $[A, B]=A B-B A$. System (2.18) is viewed as a hierarchy of non-linear equations on the infinite number of dependent variables $f_{i}(x)=f_{i}\left(x ; t_{1}, t_{2}, \ldots\right) \in \mathcal{F} * \Gamma$. When $m=1$, we have $\Gamma=\{e\}$ and $y=\partial$, in which case system (2.18) turns into the standard presentation of the KP hierarchy.

Since $L \in y+\mathcal{P}_{0}$, each equation in (2.18) can be viewed as a flow on $\mathcal{P}_{0}$. The following results are analogous to the similar standard facts about the KP hierarchy.

Proposition 2.4. (1) Equation (2.18) implies the zero-curvature equations

$$
\left[\frac{\partial}{\partial t_{k}}-\left(L^{k}\right)_{+}, \frac{\partial}{\partial t_{l}}-\left(L^{l}\right)_{+}\right]=0, \quad \forall k, l \in \mathbb{N} .
$$

(2) System (2.18) is consistent, that is, the equations of the hierarchy define commuting flows on $\mathcal{P}$.

Proofs of both statements are the same as for the usual KP hierarchy, see, e.g., (Ref. 23, Sec. 1.6).

For the usual KP hierarchy, it is customary to assume that $f_{0}=0 \mathrm{in}(2.17)$. The analogous condition in our case is as follows. For any $f \in \mathcal{F} * \Gamma$, denoted by $f_{\gamma} \in \mathcal{F}$, the coefficient in the decomposition $f=\sum_{\gamma \in \Gamma} f_{\gamma} \gamma$; in particular, $f_{e}$ denotes the coefficient at the identity element. Then our assumption on the coefficient $f_{0}$ in (2.17) will be as follows:

$$
\left(f_{0}\right)_{e}=0 .
$$

To see that this is preserved by the flows of the hierarchy, rewrite Eq. (2.18) as

$$
\frac{\partial L}{\partial t_{k}}=-\left[\left(L^{k}\right)_{-}, L\right] .
$$

Writing $\left(L^{k}\right)_{-}$as $h y^{-1}+\cdots$, we obtain that $\frac{\partial f_{0}}{\partial t_{k}}=\theta^{-1}(h)-h$, from which $\frac{\partial\left(f_{0}\right)_{e}}{\partial t_{k}}=\left(\theta^{-1}(h)-h\right)_{e}=0$, as needed.

Let us recall that for the KP hierarchy $(m=1)$, assuming $f_{0}=0$, one has $L_{+}=\partial$ and so the first equation in (2.18) gives simply that $\frac{\partial L}{\partial t_{1}}=\partial(L)$, which means that one can set $t_{1}=x$. Since in our case $f_{0}$ is nonzero, the first equation becomes more complicated and we can no longer identify $x$ with one of the time variables. Still, the zero-curvature Eq. (2.19) for all $k, l$ less than some given $n$ leads to a closed set of nonlinear equations on a finite number of coefficients of $L$. For example, to get an analogue of the usual KP equation, one can take the zero-curvature equations for all $k, l \in\{1,2,3\}$. However, the calculations become too cumbersome to be carried out explicitly.

Our main goal will be to construct some explicit solutions to this hierarchy. The solutions will be constructed in the form

$$
L=M y M^{-1}, \quad \text { where } \quad M \in 1+\mathcal{P}_{-} .
$$

Writing $M$ as $M=1+\sum_{i \geqslant 1} w_{i}(x) y^{-i}$, we can calculate $M^{-1}$, term by term, from the equation $M^{-1} M=1$,

$$
M^{-1}=1-w_{1} y^{-1}+\left(w_{1} \theta\left(w_{1}\right)-w_{2}\right) y^{-2}+\cdots .
$$


Substituting these into (2.22) gives

$$
L=y-\left(\theta^{-1}\left(w_{1}\right)-w_{1}\right)+\left(-D\left(w_{1}\right)+w_{2}-\theta^{-1}\left(w_{2}\right)-w_{1}^{2}+\theta^{-1}\left(w_{1}\right) w_{1}\right) y^{-1}+\cdots .
$$

We therefore can view (2.22) as a non-linear transformation between the variables $f_{j}$ and $w_{i}$, so that

$$
f_{0}=-\theta^{-1}\left(w_{1}\right)+w_{1}, \quad f_{1}=-D\left(w_{1}\right)+w_{2}-\theta^{-1}\left(w_{2}\right)-w_{1}^{2}+\theta^{-1}\left(w_{1}\right) w_{1},
$$

and so on. It is clear from the first formula that (2.20) is automatically satisfied.

Remark 2.5. The above $M^{-1}$ is the left inverse to $M$. In the same manner, one can construct the right inverse. By a standard argument, they coincide so $M^{-1}$ is the two-sided inverse.

The following result is straightforward.

Proposition 2.6. Suppose $M \in 1+\mathcal{P}_{-}$satisfies the equation

$$
\frac{\partial M}{\partial t_{k}}=-\left(L^{k}\right)_{-} M
$$

for some $k$, then $L=M y M^{-1}$ satisfies the equation

$$
\frac{\partial L}{\partial t_{k}}=-\left[\left(L^{k}\right)_{-}, L\right] .
$$

\section{Spherical sub-hierarchy}

Now suppose that $L$ is as above and in addition it satisfies the following property with respect to the action of the group $\Gamma$ :

$$
\epsilon_{i} L=L \epsilon_{i-1} .
$$

Then it is easy to see that such condition is only compatible with Eq. (2.18) when $k$ is divisible by $m$. Therefore, in this case, we have a sub-hierarchy consisting of the equations

$$
\frac{\partial L}{\partial t_{m j}}=\left[\left(L^{m j}\right)_{+}, L\right], \quad j \in \mathbb{N} .
$$

It is convenient to rewrite this as equations on $\widetilde{L}=L^{m}$. It follows from (2.24) that

$$
\frac{\partial \widetilde{L}}{\partial t_{m j}}=\left[\left(\widetilde{L}^{j}\right)_{+}, \widetilde{L}\right], \quad j \in \mathbb{N} .
$$

We have

$$
\widetilde{L}=y^{m}+\sum_{a>0} g_{a} y^{m-1-a}, \quad \epsilon_{i} \widetilde{L}=\widetilde{L} \epsilon_{i}, \quad g_{a} \in \mathcal{F} * \Gamma .
$$

[The fact that $g_{0}=0$ is an easy corollary of (2.20).]

We will call the hierarchies (2.25) and (2.26) the spherical sub-hierarchy. It can be further decoupled into $m$ independent hierarchies. Namely, decompose $\widetilde{L}$ as

$$
\widetilde{L}=\sum_{\ell=0}^{m-1} \widetilde{L}_{\ell}, \quad \widetilde{L}_{\ell}=\epsilon_{\ell} \widetilde{L}=\epsilon_{\ell} \widetilde{L} \epsilon_{\ell} .
$$

Then system (2.25) splits into $m$ independent systems for each of $\widetilde{L}_{\ell}$,

$$
\frac{\partial \widetilde{L}_{\ell}}{\partial t_{m j}}=\left[\left(\widetilde{L}_{\ell}^{j}\right)_{+}, \widetilde{L}_{\ell}\right], \quad \widetilde{L}_{\ell}=\epsilon_{\ell} y^{m} \epsilon_{\ell}+\sum_{a>0} \epsilon_{\ell} g_{a} y^{m-1-a} \epsilon_{\ell} .
$$

In fact, systems (2.27) with different $\ell$ are all equivalent to each other, up to a change of parameters, and if $\widetilde{L}_{0}, \ldots, \widetilde{L}_{m-1}$ are solutions to each of these hierarchies then $\widetilde{L}:=\widetilde{L}_{0}+\cdots+\widetilde{L}_{m-1}$ is a solution to $(2.25)$.

Remark 2.7. Although the cyclic quiver did not appear explicitly above, the Cherednik algebra $H_{\tau, k}\left(\mathbb{Z}_{m}\right)$ can be in fact interpreted as a deformed preprojective algebra associated with the cyclic quiver with $m$ vertices, see Sec. III B below. The role of quivers will become more apparent when we turn to constructing rational solutions of the hierarchy in Sec. IV. 


\section{DEFORMED PREPROJECTIVE ALGEBRAS AND CALOGERO-MOSER SPACES}

We begin by defining the deformed preprojective algebras due to Crawley-Boevey and Holland and by recalling some facts about their representations; the interested reader should consult Refs. 16 and 18 for details. Consider a quiver $Q$, an oriented graph with the set of vertices $I$ and arrows $Q$. For an arrow $a: i \rightarrow j$, we use the notation $t(a), h(a)$ to denote the tail $i$ and the head $j$ of $a$. Consider the double quiver $\bar{Q}$, obtained by adjoining a reverse arrow $a^{*}: j \rightarrow i$ for every arrow $a: i \rightarrow j$ in $Q$. Let $\mathbb{C} \bar{Q}$ be the path algebra of $\bar{Q}$; it has as basis the paths in $\bar{Q}$, including a trivial paths $e_{i}(i \in I)$, with multiplication given by concatenation of paths. We use the convention that for $a: i \rightarrow j$ and $b: j \rightarrow k$, the product $b a$ represents the path $i \rightarrow j \rightarrow k$ and that $b a=0$ if $h(a) \neq t(b)$.

Given $\lambda \in \mathbb{C}^{I}$, the deformed preprojective algebra $\Pi^{\lambda}(Q)$ is defined as the quotient of $\mathbb{C} \bar{Q}$ by the following relation:

$$
\sum_{a \in Q}\left(a a^{*}-a^{*} a\right)=\sum_{i \in I} \lambda_{i} e_{i}
$$

Multiplying this relation by $e_{i}$, we obtain an equivalent set of relations, one for each vertex

$$
\sum_{\substack{a \in Q \\ h(a)=i}} a a^{*}-\sum_{\substack{a \in Q \\ t(a)=i}} a^{*} a=\lambda_{i} e_{i}, \quad i \in I .
$$

For simplicity, we will simply write $\Pi^{\lambda}$ for $\Pi^{\lambda}(Q)$.

Representations of $\bar{Q}$ of dimension $\alpha \in \mathbb{N}_{0}^{I}$ are given by attaching vector spaces $V_{i}=\mathbb{C}^{\alpha_{i}}$ to each vertex $i \in I$, with the arrows of the quiver represented by linear maps $X_{a}: V_{t(a)} \rightarrow V_{h(a)}$ (with each trivial path $e_{i}$ represented by the identity map on $V_{i}$ ). The set of all representations of a given dimension is, therefore,

$$
\operatorname{Rep}(\mathbb{C} \bar{Q}, \alpha)=\bigoplus_{a \in Q} \operatorname{Mat}\left(\alpha_{h(a)} \times \alpha_{t(a)}, \mathbb{C}\right) \oplus \operatorname{Mat}\left(\alpha_{t(a)} \times \alpha_{h(a)}, \mathbb{C}\right)
$$

Isomorphism classes of representations correspond to orbits under the group

$$
G(\alpha)=\left(\prod_{i \in I} \mathrm{GL}\left(\alpha_{i}, \mathbb{C}\right)\right) / \mathbb{C}^{\times},
$$

acting by conjugation. Here $\mathbb{C}^{\times}$denotes the diagonal subgroup of scalar matrices, acting trivially on $\operatorname{Rep}(\mathbb{C} \bar{Q}, \alpha)$. Semi-simple representations correspond to closed orbits.

We have naturally $\operatorname{Rep}\left(\Pi^{\lambda}, \alpha\right) \subset \operatorname{Rep}(\mathbb{C} \bar{Q}, \alpha)$ as a subvariety cut out by relations (3.1),

$$
\sum_{\substack{a \in Q \\ h(a)=i}} X_{a} X_{a^{*}}-\sum_{\substack{a \in Q \\ t(a)=i}} X_{a^{*}} X_{a}=\lambda_{i} 1_{V_{i}}, \quad i \in I .
$$

By adding these and taking trace, we obtain

$$
\lambda \cdot \alpha=\sum_{i \in I} \lambda_{i} \alpha_{i}=0 .
$$

Consider the quotient $\operatorname{Rep}\left(\Pi^{\lambda}, \alpha\right) / / G(\alpha)$, whose points correspond to semi-simple representations of $\Pi^{\lambda}$ of dimension $\alpha$. We will be mostly interested in the situation where $\lambda$ and $\alpha$ are such that general representations in $\operatorname{Rep}\left(\Pi^{\lambda}, \alpha\right)$ are simple. In this case, the following result is known by Ref. 18, Corollary 1.4 and Lemma 6.5.

Proposition 3.1. Let $p(\alpha)=1+\sum_{a \in Q} \alpha_{t(a)} \alpha_{h(a)}-\alpha \cdot \alpha$, where $\alpha \cdot \alpha=\sum_{i \in I} \alpha_{i}^{2}$. If a general representation in $\operatorname{Re}\left(\Pi^{\lambda}, \alpha\right)$ is simple, then $\operatorname{Rep}\left(\Pi^{\lambda}, \alpha\right) / / G(\alpha)$ is a reduced and irreducible scheme of dimension $2 p(\alpha)$, smooth at the points corresponding to (the isomorphism classes of) simple representations. In particular, if all representations in $\operatorname{Rep}\left(\Pi^{\lambda}, \alpha\right)$ are simple, then $\operatorname{Rep}\left(\Pi^{\lambda}, \alpha\right) / / G(\alpha)$ is a smooth connected affine variety of dimension $2 p(\alpha)$.

Let us remark that the varieties $\operatorname{Rep}\left(\Pi^{\lambda}, \alpha\right) / / G(\alpha)$ can be obtained by Hamiltonian reduction. ${ }^{40}$ Indeed, the space $\operatorname{Rep}(\mathbb{C} \bar{Q}, \alpha)$ has a natural complex symplectic structure

$$
\omega=\sum_{a \in Q} \operatorname{tr}\left(\mathrm{d} X_{a^{*}} \wedge \mathrm{d} X_{a}\right)
$$


invariant under $G(\alpha)$. The left-hand side of (3.3) can then be viewed as a component $p_{i}$ of a moment map $\mu_{\alpha}$ for the action of $G(\alpha)$ on $\operatorname{Rep}(\mathbb{C} \bar{Q}, \alpha)$. Therefore, we can view $\operatorname{Rep}\left(\Pi^{\lambda}, \alpha\right)$ as a fibre $\mu_{\alpha}^{-1}(\bar{\lambda})$ of the moment map at $\bar{\lambda}=\sum_{i \in I} \lambda_{i} 1_{V_{i}}$,

$$
\operatorname{Rep}\left(\Pi^{\lambda}, \alpha\right) / / G(\alpha)=\mu_{\alpha}^{-1}(\bar{\lambda}) / / G(\alpha) .
$$

As a consequence, in the situation when $G(\alpha)$ acts freely, quotient (3.6) carries a natural symplectic structure.

\section{A. Quiver varieties}

Let $Q_{0}$ be an arbitrary quiver with the vertex set $I$. A framing of $Q_{0}$ is a quiver $Q$ that has one additional vertex, denoted $\infty$, and a number of arrows $i \rightarrow \infty$ from the vertices of $Q_{0}$ (multiple arrows are allowed). Given $\alpha \in \mathbb{N}_{0}^{I}$ and $\lambda \in \mathbb{C}^{I}$, we extend them from $Q_{0}$ to $Q$ by putting $\alpha_{\infty}=1$ and $\lambda_{\infty}=-\lambda \cdot \alpha$. Thus, we put

$$
\boldsymbol{\alpha}=(1, \alpha), \quad \boldsymbol{\lambda}=(-\lambda \cdot \alpha, \lambda) .
$$

This choice ensures that $\boldsymbol{\lambda} \cdot \boldsymbol{\alpha}=0$. Consider the space $\operatorname{Rep}\left(\Pi^{\lambda}, \boldsymbol{\alpha}\right)$ of representations of the deformed preprojective algebra of $Q$ of dimension $\alpha$. The quotients

$$
\mathfrak{M}_{\alpha, \lambda}(Q):=\operatorname{Rep}\left(\Pi^{\lambda}, \boldsymbol{\alpha}\right) / / G(\boldsymbol{\alpha})
$$

are closely related to Nakajima quiver varieties, see Ref. 18, pp. 261-262. Note that since $\alpha_{\infty}=1$, we have

$$
G(\boldsymbol{\alpha})=\left(\prod_{i \in I \sqcup\{\infty\}} \mathrm{GL}\left(\alpha_{i}, \mathbb{C}\right)\right) / \mathbb{C}^{\times} \simeq \mathrm{GL}(\alpha):=\prod_{i \in I} \mathrm{GL}\left(\alpha_{i}, \mathbb{C}\right),
$$

where the group on the right-hand side acts on $\bigoplus_{i \in I} V_{i}$.

Recall that Kac defines in Ref. 35 the root system $\Delta(Q)$ for an arbitrary quiver $Q$. Let us say that $\lambda \in \mathbb{C}^{I}$ is regular if $\lambda \cdot \alpha \neq 0$ for any $\alpha \in \Delta\left(Q_{0}\right)$. Then the following result is a corollary of Ref. 18, Theorem 1.2 (cf. Ref. 12, Proposition 3):

Proposition 3.2. Let $\alpha$ and $\lambda$ be as in (3.7), with $\lambda$ regular. Choose a framing $Q$ of $Q_{0}$ and let $\Pi^{\lambda}=\Pi^{\lambda}(Q)$. Then there is $a \Pi^{\lambda}$-module of dimension $\alpha$ if and only if $\alpha \in \Delta_{+}(Q)$, where $\Delta_{+}(Q)$ is the set of positive roots of $Q$. Moreover, every such module is simple.

Combining this with Proposition 3.1, we conclude that for $(1, \alpha) \in \Delta_{+}(Q)$ and regular $\lambda \in \mathbb{C}^{I}$, the variety $\mathfrak{M}_{\alpha, \lambda}(Q)$ is smooth, of dimension $2 p(\boldsymbol{\alpha})$. Note that for imaginary roots $\boldsymbol{\alpha}$, one has $p(\boldsymbol{\alpha})$ $>0$, while for real roots $p(\boldsymbol{\alpha})=0$. Furthermore, if $\boldsymbol{\alpha}$ is a real positive root, then there is a unique representation of $\Pi^{\lambda}$ of dimension $\alpha$ up to isomorphism (Ref. 19, Proposition 1.2). This means that in this case, $\mathfrak{M}_{\alpha, \lambda}(Q)$ consists of one point.

\section{B. Case of a cyclic quiver}

Now let $Q_{0}$ be a cyclic oriented quiver with the set of vertices $I=\mathbb{Z} / m \mathbb{Z}=\{0,1, \ldots, m-1\}$ and $m$ arrows $a_{i}: i+1 \rightarrow i$. The double quiver $\bar{Q}_{0}$ is obtained by adding the reverse arrows $a_{i}^{*}: i \rightarrow i+1$. For $\lambda \in \mathbb{C}^{I}$, the deformed preprojective algebra $\Pi^{\lambda}$ is the quotient of $\mathbb{C} \bar{Q}_{0}$ by the relations

$$
a_{i} a_{i}^{*}-a_{i-1}^{*} a_{i-1}=\lambda_{i} e_{i}, \quad i \in I .
$$

If $m=1$, then we have a one-loop quiver $Q_{0}$, so $\Pi^{\lambda}$ in this case is isomorphic to $\mathbb{C}\left\langle a, a^{*}\right\rangle /\left\{a a^{*}-a^{*} a=\lambda\right\}$. For $m>1$, there is an isomorphism between $\Pi^{\lambda}$ and Cherednik algebra $H_{\tau, k}\left(\mathbb{Z}_{m}\right)$, given by

$$
a_{i} \mapsto \epsilon_{i} x, \quad a_{i}^{*} \mapsto y \epsilon_{i}, \quad e_{i} \mapsto \epsilon_{i},
$$

with the parameters $\lambda_{i}$ and $\tau, k$ related by (2.5).

\section{Calogero-Moser spaces}

Let $Q_{0}$ be a cyclic oriented quiver with the set of vertices $I=\mathbb{Z} / m \mathbb{Z}$ as above. Calogero-Moser spaces are the varieties (3.8) associated with certain framings of $Q_{0}$. We will consider one of the 
following framings:

$$
\begin{array}{ll}
Q=Q_{0} \sqcup\left\{b_{i, \alpha}: i \rightarrow \infty \mid i \in I, \alpha=1, \ldots, d\right\}, & d \in \mathbb{N}, \\
Q=Q_{0} \sqcup\left\{b_{\ell, \alpha}: \ell \rightarrow \infty \mid \alpha=1, \ldots, d\right\}, & \ell \in I, \quad d \in \mathbb{N}, \\
Q=Q_{0} \sqcup\left\{b_{i}: i \rightarrow \infty \mid i \in I\right\}, & \\
Q=Q_{0} \sqcup\left\{b_{\ell}: \ell \rightarrow \infty\right\}, & \ell \in I .
\end{array}
$$

Quivers (3.12) and (3.13) have $m d$ and $d$ added arrows, respectively; they will only be needed in Sec. VI. The other two quivers correspond to $d=1$.

For $\lambda=\left(\lambda_{0}, \ldots, \lambda_{m-1}\right) \in \mathbb{C}^{m}$ and $\alpha=\left(\alpha_{0}, \ldots, \alpha_{m-1}\right) \in \mathbb{N}_{0}^{m}$, we put $\alpha=(1, \alpha)$ and $\boldsymbol{\lambda}=(-\lambda \cdot \alpha, \lambda)$ and consider representations of $\Pi^{\lambda}$ of dimension $\alpha$. Each representation consists of a vector space

$$
V_{\infty} \oplus V_{0} \oplus \cdots \oplus V_{m-1}, \quad \text { where } V_{\infty}=\mathbb{C}, \quad V_{i}=\mathbb{C}^{\alpha_{i}}, i \in I,
$$

together with a collection of linear maps representing the arrows of $\bar{Q}$, which should satisfy relations (3.3). It is more convenient to consider representations of $\left(\Pi^{\lambda}\right)^{\text {opp }}$, i.e., right $\Pi^{\lambda}$-modules (this is in agreement with the conventions in Ref. 12). Therefore, analogously to (3.8) and (3.9), we define the Calogero-Moser space associated with a framing $Q$ of $Q_{0}$ as

$$
\mathcal{C}_{\alpha, \lambda}(Q)=\operatorname{Rep}\left(\left(\Pi^{\lambda}\right)^{\text {opp }}, \boldsymbol{\alpha}\right) / / \mathrm{GL}(\alpha), \quad \alpha \in \mathbb{N}_{0}^{m}, \quad \lambda \in \mathbb{C}^{m} .
$$

Since representations of $\left(\Pi^{\lambda}\right)^{\text {opp }}$ can be realised as dual to those of $\Pi^{\lambda}$, all the properties of $\operatorname{Rep}\left(\Pi^{\lambda}, \alpha\right)$ discussed earlier remain valid in this setting. In particular, for regular $\lambda$, the varieties $\mathcal{C}_{\alpha, \lambda}$ are smooth. Recall that, for a cyclic quiver $Q_{0}$, the root system $\Delta\left(Q_{0}\right) \subset \mathbb{Z}^{m}$ is isomorphic to the affine root system of type $\widetilde{A}_{m-1}$. Under this isomorphism, the roots in $\Delta\left(Q_{0}\right)$ are obtained by writing the roots of $\widetilde{A}_{m-1}$ in the basis of simple roots $\varepsilon_{0}, \ldots, \varepsilon_{m-1}$. In particular, imaginary roots $n \delta=n\left(\varepsilon_{0}+\cdots+\varepsilon_{m-1}\right)$ correspond to $\alpha=(n, \ldots, n) \in \Delta\left(Q_{0}\right)$. The real roots are of the form $n \delta \pm\left(\varepsilon_{i}+\cdots+\varepsilon_{j-1}\right)$ with $1 \leqslant i<j \leqslant m-1$. Therefore, $\lambda \in \mathbb{C}^{m}$ is regular iff

$$
\lambda_{0}+\cdots+\lambda_{m-1} \neq 0, \quad n\left(\lambda_{0}+\cdots+\lambda_{m-1}\right) \neq \lambda_{i}+\cdots+\lambda_{j-1},
$$

for all $n \in \mathbb{Z}$ and $1 \leqslant i<j \leqslant m-1$. Assuming that $\lambda_{i}$ are the same as in (2.5), we conclude that the parameters $(\tau, k) \in \mathbb{C}^{m+1}$ are regular iff $\tau \neq 0$ and

$$
\frac{k_{i}-k_{j}}{\tau} \notin \frac{j-i}{m}+\mathbb{Z} \quad \text { for } \quad 0 \leqslant i \neq j \leqslant m-1 .
$$

Below we will assume that the parameters are regular, unless specified otherwise.

Let us describe the varieties $\mathcal{C}_{\alpha, \lambda}$ more concretely. We start by taking $Q$ as in (3.14). A right action of $\Pi^{\lambda}$ on a vector space (3.16) consists of a collection of maps

$$
X_{a_{i}}: V_{i} \rightarrow V_{i+1}, \quad X_{a_{i}^{*}}: V_{i+1} \rightarrow V_{i}, \quad X_{b_{i}}: \mathbb{C} \rightarrow V_{i}, \quad X_{b_{i}^{*}}: V_{i} \rightarrow \mathbb{C} .
$$

Note that the maps go in the opposite direction to arrows because we are considering representations of the opposite algebra. To simplify the notation, put

$$
X_{i}:=X_{a_{i}}, \quad Y_{i}:=X_{a_{i}^{*}}, \quad v_{i}:=X_{b_{i}}, \quad w_{i}:=X_{b_{i}^{*}} .
$$

Relations (3.1) then read as follows:

$$
\begin{aligned}
Y_{i} X_{i}-X_{i-1} Y_{i-1}-v_{i} w_{i} & =\lambda_{i} 1_{V_{i}} \quad(i \in \mathbb{Z} / m \mathbb{Z}), \\
\sum_{i \in I} w_{i} v_{i} & =\lambda_{\infty} .
\end{aligned}
$$

Note that since we are assuming that $\lambda_{\infty}=-\lambda \cdot \alpha$, the last relation follows from (3.21) automatically by adding up and taking traces. Recall that the points of $\mathcal{C}_{\alpha, \lambda}$ are the equivalence classes of the above data $X_{i}, Y_{i}, v_{i}, w_{i}$ under the action of $\mathrm{GL}(\alpha)$.

It will be convenient to put $V=\bigoplus_{i \in I} V_{i}$ and denote the projection onto the $i$ th direct summand in $V$ as $e_{i}$, so $\left.e_{i}\right|_{V_{j}}=\delta_{i, j} 1_{V_{i}}$. Let us extend maps (3.20) up to $X_{i}, Y_{i}: V \rightarrow V, v_{i}: \mathbb{C} \rightarrow V$, and $w_{i}: V \rightarrow \mathbb{C}$ in an obvious way (e.g., $\left.Y_{i}\right|_{V_{j}}=0$ for $j \neq i+1$ ) and put

$$
X=\sum_{i \in I} X_{i}, \quad Y=\sum_{i \in I} Y_{i}, \quad v=\sum_{i \in I} v_{i}, \quad w=\sum_{i \in I} w_{i} .
$$


Then (3.21) is equivalent to

$$
Y X-X Y-\sum_{i \in I} v_{i} w_{i}=\sum_{i \in I} \lambda_{i} e_{i}
$$

and we have

$$
e_{i+1} X=X e_{i}=X_{i}, \quad e_{i} Y=Y e_{i+1}=Y_{i}, \quad e_{i} v=v_{i}, \quad w e_{i}=w_{i} .
$$

In this notation, the action of $g \in \mathrm{GL}(\alpha) \subset \operatorname{End}(V)$ sends $X, Y, v, w$ to

$$
g X g^{-1}, \quad g Y g^{-1}, \quad g v, \quad w g^{-1} \text {. }
$$

This action is free when $\lambda$ is regular, and its orbits are identified with the points of the Calogero-Moser space $\mathcal{C}_{\alpha, \lambda}$, whose dimension of which is given by Proposition 3.1. In our case, the set of vertices of $Q$ is $I \sqcup\{\infty\}$ and the dimension vector is $\alpha=(1, \alpha)$. We have

$$
p(\boldsymbol{\alpha})=1+\sum_{i \in I} \alpha_{i} \alpha_{i+1}+\sum_{i \in I} \alpha_{i}-1-\sum_{i \in I} \alpha_{i}^{2}=\sum_{i \in I} \alpha_{i}-\frac{1}{2} \sum_{i \in I}\left(\alpha_{i}-\alpha_{i+1}\right)^{2} .
$$

Therefore,

$$
\operatorname{dim} \mathcal{C}_{\alpha, \lambda}=2 \sum_{i \in I} \alpha_{i}-\sum_{i \in I}\left(\alpha_{i}-\alpha_{i+1}\right)^{2} .
$$

In the case of quiver (3.15), everything looks similar except that we only have one pair $v_{\ell}, w_{\ell}$; therefore, we may just put $v_{i}=w_{i}=0$ for $i \neq \ell$. This turns Eqs. (3.21) and (3.22) into

$$
\begin{aligned}
Y_{i} X_{i}-X_{i-1} Y_{i-1}-\delta_{i, \ell} v_{\ell} w_{\ell} & =\lambda_{i} 1_{V_{i}} \quad(i \in \mathbb{Z} / m \mathbb{Z}), \\
w_{\ell} v_{\ell} & =\lambda_{\infty} .
\end{aligned}
$$

Relation (3.24) becomes

$$
Y X-X Y-v_{\ell} w_{\ell}=\sum_{i \in I} \lambda_{i} e_{i}
$$

and (3.25) and (3.26) remain the same but with $v_{i}=w_{i}=0$ for $i \neq \ell$. Since in this case, $Q$ has only one arrow going to $\infty$, we find that

$$
\operatorname{dim} \mathcal{C}_{\alpha, \lambda}=2 \alpha_{\ell}-\sum_{i \in I}\left(\alpha_{i}-\alpha_{i+1}\right)^{2},
$$

assuming that $(1, \alpha)$ is a positive root for $Q$ and $\lambda$ is regular.

\section{RATIONAL SOLUTIONS VIA CALOGERO-MOSER SPACES}

In this section, we construct rational solutions of the hierarchy, parametrised by points of the suitable Calogero-Moser spaces. We start by setting up the notation and doing some preliminary calculations.

\section{A. Preliminary calculations}

Let $Q$ be quiver (3.14) and $\mathcal{C}_{\alpha, \lambda}$ be space (3.17). Take a point of $\mathcal{C}_{\alpha, \lambda}$; it is represented by (isomorphism class of) data $X, Y, v, w$ satisfying (3.24) and (3.25). Put

$$
M=1-\sum_{i, j \in I} \epsilon_{i} w_{i}\left(X-x 1_{V}\right)^{-1}\left(Y-y 1_{V}\right)^{-1} v_{j} \epsilon_{j} .
$$

Here we regard $\left(X-x 1_{V}\right)^{-1}$ and $\left(Y-y 1_{V}\right)^{-1}$ as elements of the tensor product $\mathcal{P} \otimes$ End $V$ by expanding $\left(y 1_{V}-Y\right)^{-1}$ into $\sum_{k \geqslant 0} Y^{k} y^{-k-1}$. Similarly, $e_{i}$ and $\epsilon_{i}$ are identified with the elements $1 \otimes e_{i}$ and $\epsilon_{i} \otimes 1_{V}$ so that $e_{i} \epsilon_{i}=\epsilon_{i} e_{i}=\epsilon_{i} \otimes e_{i}$, while $v_{i}$ and $w_{i}$ become maps between $\mathcal{P} \otimes V$ and $\mathcal{P}$. Therefore $M$ is an element of $\mathcal{P}$. It is clear that $M$ is invariant under transformations (3.26). Thus, (4.1) defines a map $\mathcal{C}_{\alpha, \lambda} \rightarrow 1+\mathcal{P}_{-}$.

Lemma 4.1. The inverse of $M$ is given by

$$
M^{-1}=1+\sum_{i, j \in I} \epsilon_{i} w_{i}\left(Y-y 1_{V}\right)^{-1}\left(X-x 1_{V}\right)^{-1} v_{j} \epsilon_{j}
$$


Proof. We need to check that $M^{-1} M=1$. Introduce the following shorthand notation:

$$
\widehat{X}=X-x 1_{V}, \quad \widehat{Y}=Y-y 1_{V} .
$$

It easily follows from (2.5) and (3.24) that

$$
\sum_{i \in I} v_{i} w_{i}=\widehat{Y} \widehat{X}-\widehat{X} \widehat{Y}+\sum_{i \in I} \lambda_{i}\left(\epsilon_{i}-e_{i}\right) .
$$

Next, note the following identities:

$$
\begin{aligned}
& e_{a} \epsilon_{b} \widehat{X}^{-1} \widehat{Y}^{-1} e_{c} \epsilon_{d}=0, \quad \text { if } a-c \neq b-d, \\
& e_{a} \epsilon_{a} \widehat{X}^{-1} \widehat{Y}^{-1} e_{c} \epsilon_{c}=\epsilon_{a} e_{a} \widehat{X}^{-1} \widehat{Y}^{-1} e_{c}, \quad e_{c} \epsilon_{c} \widehat{X}^{-1} \widehat{Y}^{-1} e_{a} \epsilon_{a}=e_{c} \widehat{X}^{-1} \widehat{Y}^{-1} e_{a} \epsilon_{a}, \\
& e_{a} \epsilon_{a} \widehat{X}^{-1} \widehat{Y}^{-1}\left(\epsilon_{c}-e_{c}\right)=0, \quad\left(\epsilon_{c}-e_{c}\right) \widehat{X}^{-1} \widehat{Y}^{-1} e_{a} \epsilon_{a}=0 .
\end{aligned}
$$

Here $a, b, c, d \in I=\mathbb{Z} / m \mathbb{Z}$. To prove (4.4), note that

$$
\begin{aligned}
e_{a} \epsilon_{b} \widehat{X}^{-1} \widehat{Y}^{-1} e_{c} \epsilon_{d} & =\sum_{i, j \geqslant 0} e_{a} \epsilon_{b} X^{i} Y^{j} x^{-1-i} y^{-1-j} e_{c} \epsilon_{d} \\
& =\sum_{i, j \geqslant 0} e_{a} \epsilon_{b} e_{c+i-j} \epsilon_{d+i-j} X^{i} Y^{j} x^{-1-i} y^{-1-j},
\end{aligned}
$$

which is obviously zero if $a-c \neq b-d$ [here we used (2.4) and (3.25)]. Formula (4.5) follows from (4.4) by substituting $e_{c}=\sum_{i \in I} e_{c} \epsilon_{i}$ to the right hand side of (4.5). Finally, to derive (4.6), one replaces $\epsilon_{c}-e_{c}$ by $\sum_{i \in I}\left(e_{i} \epsilon_{c}-e_{c} \epsilon_{i}\right)$ and uses (4.4).

Below we will repeatedly use the following corollary of (4.5):

$$
\epsilon_{k} w_{k} \widehat{X}^{-1} \widehat{Y}^{-1} v_{j} \epsilon_{j}=w_{k} \widehat{X}^{-1} \widehat{Y}^{-1} v_{j} \epsilon_{j}=\epsilon_{k} w_{k} \widehat{X}^{-1} \widehat{Y}^{-1} v_{j}
$$

In other words, one can remove either $\epsilon_{k}$ or $\epsilon_{j}$ in this expression.

Now, using the formulas for $M$ and $M^{-1}$, we get

$$
\begin{aligned}
M^{-1} M= & 1-\sum_{i, j} \epsilon_{i} w_{i} \widehat{X}^{-1} \widehat{Y}^{-1} v_{j} \epsilon_{j}+\sum_{i, j} \epsilon_{i} w_{i} \widehat{Y}^{-1} \widehat{X}^{-1} v_{j} \epsilon_{j} \\
& -\sum_{i, j, k} \epsilon_{i} w_{i} \widehat{Y}^{-1} \widehat{X}^{-1} v_{k} \epsilon_{k} w_{k} \widehat{X}^{-1} \widehat{Y}^{-1} v_{j} \epsilon_{j} .
\end{aligned}
$$

We can use (4.7) and replace the last sum by

$$
\sum_{i, j} \epsilon_{i} w_{i} \widehat{Y}^{-1} \widehat{X}^{-1}\left(\sum_{k} v_{k} w_{k}\right) \widehat{X}^{-1} \widehat{Y}^{-1} v_{j} \epsilon_{j} .
$$

Replacing $\sum_{k} v_{k} w_{k}$ by (4.3) and substituting back in (4.8), we obtain

$$
M^{-1} M=1-\sum_{i, j, k \in I} \lambda_{k} \epsilon_{i} w_{i} \widehat{Y}^{-1} \widehat{X}^{-1}\left(\epsilon_{k}-e_{k}\right) \widehat{X}^{-1} \widehat{Y}^{-1} v_{j} \epsilon_{j} .
$$

By (4.6),

$$
\left(\epsilon_{k}-e_{k}\right) \widehat{X}^{-1} \widehat{Y}^{-1} v_{j} \epsilon_{j}=\left(\epsilon_{k}-e_{k}\right) \widehat{X}^{-1} \widehat{Y}^{-1} e_{j} \epsilon_{j} v_{j}=0 .
$$

We conclude that $M^{-1} M=1$, as needed.

Lemma 4.2. Let $L=M y M^{-1}$. For $k \in \mathbb{N}$, we have

$$
\begin{aligned}
\left(L^{k}\right)_{-}= & \sum_{i, j} \epsilon_{i} w_{i-k} Y^{k} \widehat{Y}^{-1} \widehat{X}^{-1} v_{j} \epsilon_{j}-\sum_{i, j} \epsilon_{i} w_{i} \widehat{X}^{-1} \widehat{Y}^{-1} Y^{k} v_{j+k} \epsilon_{j} \\
& -\sum_{i, j, \ell} \epsilon_{i} w_{i} \widehat{X}^{-1} \widehat{Y}^{-1} Y^{k} v_{\ell} w_{\ell-k} \widehat{Y}^{-1} \widehat{X}^{-1} v_{j} \epsilon_{j} \\
& +\sum_{i, j, \ell} \sum_{a=0}^{k-1} \epsilon_{i} w_{i} \widehat{X}^{-1} Y^{a} v_{\ell} w_{\ell-k} Y^{k-1-a} \widehat{Y}^{-1} \widehat{X}^{-1} v_{j} \epsilon_{j} .
\end{aligned}
$$


Proof. Write $M, M^{-1}$ as

$$
M=1-\sum_{i, j} \mu_{i j}, \quad M^{-1}=1+\sum_{i, j} \tilde{\mu}_{i j},
$$

where

$$
\mu_{i j}=\epsilon_{i} w_{i} \widehat{X}^{-1} \widehat{Y}^{-1} v_{j} \epsilon_{j}, \quad \tilde{\mu}_{i j}=\epsilon_{i} w_{i} \widehat{Y}^{-1} \widehat{X}^{-1} v_{j} \epsilon_{j} .
$$

Therefore,

$$
L^{k}=M y^{k} M^{-1}=y^{k}+\sum_{i, j} y^{k} \tilde{\mu}_{i j}-\sum_{i, j} \mu_{i j} y^{k}-\sum_{i j i^{\prime} j^{\prime}} \mu_{i j} y^{k} \tilde{\mu}_{i^{\prime} j^{\prime}} .
$$

Taking into account that $\epsilon_{j} y^{k}=y^{k} \epsilon_{j-k}$, one gets

$$
\begin{aligned}
\sum_{i, j} y^{k} \tilde{\mu}_{i j} & =\sum_{i, j} \epsilon_{i} w_{i-k} y^{k} \widehat{Y}^{-1} \widehat{X}^{-1} v_{j} \epsilon_{j}, \\
-\sum_{i, j} \mu_{i j} y^{k} & =-\sum_{i, j} \epsilon_{i} w_{i} \widehat{X}^{-1} \widehat{Y}^{-1} y^{k} v_{j+k} \epsilon_{j}, \\
-\sum_{i, j, i^{\prime} j^{\prime}} \mu_{i j} y^{k} \tilde{\mu}_{i^{\prime} j^{\prime}} & =-\sum_{i, j, \ell} \epsilon_{i} w_{i} \widehat{X}^{-1} \widehat{Y}^{-1} \epsilon_{\ell} v_{\ell} y^{k} w_{\ell-k} \widehat{Y}^{-1} \widehat{X}^{-1} v_{j} \epsilon_{j} .
\end{aligned}
$$

By (4.7), we can remove $\epsilon_{\ell}$ in the last formula to get

$$
-\sum_{i, j, i^{\prime}, j^{\prime}} \mu_{i j} y^{k} \tilde{\mu}_{i^{\prime} j^{\prime}}=-\sum_{i, j, \ell} \epsilon_{i} w_{i} \widehat{X}^{-1} \widehat{Y}^{-1} y^{k} v_{\ell} w_{\ell-k} \widehat{Y}^{-1} \widehat{X}^{-1} v_{j} \epsilon_{j} .
$$

From an elementary formula $Y^{k}-y^{k} 1_{V}=\left(Y-y 1_{V}\right) \sum_{a=0}^{k-1} y^{k-1-a} Y^{a}$, we obtain

$$
y^{k} \widehat{Y}^{-1}=\widehat{Y}^{-1} y^{k}=\widehat{Y}^{-1} Y^{k}-\sum_{a=0}^{k-1} Y^{a} y^{k-1-a}, \quad\left(y^{k} \widehat{Y}^{-1}\right)_{-}=Y^{k} \widehat{Y}^{-1} .
$$

Similarly,

$$
\widehat{Y}^{-1} y^{k} v_{\ell} w_{\ell-k} \widehat{Y}^{-1}=\widehat{Y}^{-1} Y^{k} v_{\ell} w_{\ell-k} \widehat{Y}^{-1}-\sum_{a=0}^{k-1} Y^{a} v_{\ell} w_{\ell-k} y^{k-1-a} \widehat{Y}^{-1},
$$

and by applying formula (4.15), we obtain

$$
\left(\widehat{Y}^{-1} y^{k} v_{\ell} w_{\ell-k} \widehat{Y}^{-1}\right)_{-}=\widehat{Y}^{-1} Y^{k} v_{\ell} w_{\ell-k} \widehat{Y}^{-1}-\sum_{a=0}^{k-1} Y^{a} v_{\ell} w_{\ell-k} Y^{k-1-a} \widehat{Y}^{-1} .
$$

Using these facts, we compute the negative part of (4.12)-(4.14) which results in (4.10).

\section{B. Solutions for the hierarchy}

Let $Q$ be quiver (3.14) and $\boldsymbol{\alpha}=(1, \alpha)$ with $\alpha \in \mathbb{N}_{0}^{I}$. Consider $\operatorname{Rep}\left(\mathbb{C} \bar{Q}^{\text {opp }}, \boldsymbol{\alpha}\right)$; a point of $\operatorname{Rep}\left(\mathbb{C} \bar{Q}^{\text {opp }}, \boldsymbol{\alpha}\right)$ is a collection of maps

$$
X_{i}: V_{i} \rightarrow V_{i+1}, \quad Y_{i}: V_{i+1} \rightarrow V_{i}, \quad v_{i}: \mathbb{C} \rightarrow V_{i}, \quad w_{i}: V_{i} \rightarrow \mathbb{C} .
$$

Thus, this is an affine space of dimension $2 \sum_{i \in I}\left(\alpha_{i} \alpha_{i+1}+\alpha_{i}\right)$. It has a natural symplectic structure (3.5),

$$
\omega=\sum_{i \in I}\left(\operatorname{tr}\left(\mathrm{d} Y_{i} \wedge \mathrm{d} X_{i}\right)+\mathrm{d} w_{i} \wedge \mathrm{d} v_{i}\right)
$$

The corresponding Poisson bracket is given by

$$
\left\{\left(X_{i}\right)_{a b},\left(Y_{j}\right)_{c d}\right\}=\delta_{i j} \delta_{a d} \delta_{b c}, \quad\left\{\left(v_{i}\right)_{a},\left(w_{j}\right)_{b}\right\}=\delta_{i j} \delta_{a b} .
$$

(All remaining brackets are zero.) 
As before, we will combine $X_{i}, Y_{j}, v_{i}, w_{i}$ into $X, Y \in \operatorname{End} V$ and $v: \mathbb{C} \rightarrow V, w: \mathbb{C} \rightarrow V$, see (3.23). For $k \in \mathbb{N}$, define a flow on $\operatorname{Rep}\left(\mathbb{C} \bar{Q}^{\text {opp }}, \boldsymbol{\alpha}\right)$ by the formulas

$$
\begin{aligned}
\dot{X} & =-\sum_{\ell \in I} \sum_{a=0}^{k-1} Y^{a} v_{\ell} w_{\ell-k} Y^{k-a-1}, \quad \dot{Y}=0, \\
\dot{v} & =-Y^{k} v, \quad \dot{w}=w Y^{k},
\end{aligned}
$$

where the dot denotes the derivative with respect to the time variable $t_{k}$. Equivalently,

$$
\begin{aligned}
\dot{X}_{i} & =-\sum_{a=0}^{k-1} Y_{i+1} Y_{i+2} \cdots Y_{i+a} v_{i+a+1} w_{i+a+1-k} Y_{i+a+1-k} \cdots Y_{i-1}, \\
\dot{Y}_{i} & =0 \\
\dot{v}_{i} & =-Y^{k} v_{i+k}=-Y_{i} Y_{i+1} \cdots Y_{i+k-1} v_{i+k}, \\
\dot{w}_{i} & =w_{i-k} Y^{k}=w_{i-k} Y_{i-k} Y_{i-k+1} \cdots Y_{i-1} .
\end{aligned}
$$

It is easy to check that this is a Hamiltonian flow, with the Hamiltonian

$$
H_{k}=-w Y^{k} v=-\sum_{i \in I} w_{i} Y_{i} \ldots Y_{i+k-1} v_{i+k} .
$$

The Hamiltonian $H_{k}$ is invariant under the action (3.26) of $G(\boldsymbol{\alpha})$; therefore, the moment map is a first integral, thus the flow descends to a Hamiltonian flow on a symplectic quotient $\mathcal{C}_{\alpha, \lambda}$. [This can also be checked directly by differentiating relations (3.21) and (3.22).]

Proposition 4.3. Let $L=M y M^{-1}$ where $M$ and $M^{-1}$ are as in (4.1) and (4.2). If $X, Y, v, w$ satisfy Eqs. (4.18) and (4.19), then $L$ satisfies $\dot{L}=\left[\left(L^{k}\right)_{+}, L\right]$.

Proof. According to Proposition 2.6, it suffices to check that

$$
\dot{M}=-\left(L^{k}\right) \_M .
$$

Let us start by calculating the right-hand side. Note that the second and third sum in (4.10) are nothing but

$$
\left(-\sum_{i, j} \epsilon_{i} w_{i} \widehat{X}^{-1} \widehat{Y}^{-1} Y^{k} v_{j+k} \epsilon_{j}\right) M^{-1} .
$$

Therefore, substituting them in the right-hand side of (4.25) results in

$$
\sum_{i, j} \epsilon_{i} w_{i} \widehat{X}^{-1} \widehat{Y}^{-1} Y^{k} v_{j+k} \epsilon_{j}
$$

Multiplying the remaining terms in (4.10) by $M$ gives

$$
\begin{gathered}
\left(\sum_{i, j} \epsilon_{i} w_{i-k} Y^{k} \widehat{Y}^{-1} \widehat{X}^{-1} v_{j} \epsilon_{j}+\sum_{i, j, \ell} \sum_{a=0}^{k-1} \epsilon_{i} w_{i} \widehat{X}^{-1} Y^{a} v_{\ell} w_{\ell-k} Y^{k-1-a} \widehat{Y}^{-1} \widehat{X}^{-1} v_{j} \epsilon_{j}\right) M \\
=\sum_{i, j} \epsilon_{i} w_{i-k} Y^{k} \widehat{Y}^{-1} \widehat{X}^{-1} v_{j} \epsilon_{j}+\sum_{i, j, \ell} \sum_{a=0}^{k-1} \epsilon_{i} w_{i} \widehat{X}^{-1} Y^{a} v_{\ell} w_{\ell-k} Y^{k-1-a} \widehat{Y}^{-1} \widehat{X}^{-1} v_{j} \epsilon_{j} \\
-\sum_{i, j, r} \epsilon_{i} w_{i-k} Y^{k} \widehat{Y}^{-1} \widehat{X}^{-1} v_{r} w_{r} \widehat{X}^{-1} \widehat{Y}^{-1} v_{j} \epsilon_{j} \\
-\sum_{i, j, \ell, r} \sum_{a=0}^{k-1} \epsilon_{i} w_{i} \widehat{X}^{-1} Y^{a} v_{\ell} w_{\ell-k} Y^{k-1-a} \widehat{Y}^{-1} \widehat{X}^{-1} v_{r} w_{r} \widehat{X}^{-1} \widehat{Y}^{-1} v_{j} \epsilon_{j} .
\end{gathered}
$$

Replacing $\sum_{r} v_{r} w_{r}$ in the last two terms by (4.3) and applying (4.6) and (4.7), we are left after cancellations with the following terms:

$$
\sum_{i, j} \epsilon_{i} w_{i-k} Y^{k} \widehat{X}^{-1} \widehat{Y}^{-1} v_{j} \epsilon_{j}+\sum_{i, j, \ell} \sum_{a=0}^{k-1} \epsilon_{i} w_{i} \widehat{X}^{-1} Y^{a} v_{\ell} w_{\ell-k} Y^{k-1-a} \widehat{X}^{-1} \widehat{Y}^{-1} v_{j} \epsilon_{j}
$$


Now, the right-hand side of (4.25) is obtained by subtracting (4.28) from (4.26), so

$$
\begin{aligned}
-\left(L^{k}\right)_{-} M= & \sum_{i, j} \epsilon_{i} w_{i} \widehat{X}^{-1} \widehat{Y}^{-1} Y^{k} v_{j+k} \epsilon_{j}-\sum_{i, j} \epsilon_{i} w_{i-k} Y^{k} \widehat{X}^{-1} \widehat{Y}^{-1} v_{j} \epsilon_{j} \\
& -\sum_{i, j, \ell} \sum_{a=0}^{k-1} \epsilon_{i} w_{i} \widehat{X}^{-1} Y^{a} v_{\ell} w_{\ell-k} Y^{k-1-a} \widehat{X}^{-1} \widehat{Y}^{-1} v_{j} \epsilon_{j} .
\end{aligned}
$$

On the other hand, from the formula for $M$, we get that

$$
\dot{M}=-\sum_{i, j} \epsilon_{i} w_{i} \widehat{X}^{-1} \widehat{Y}^{-1} \dot{v}_{j} \epsilon_{j}-\sum_{i, j} \epsilon_{i} \dot{w}_{i} \widehat{X}^{-1} \widehat{Y}^{-1} v_{j} \epsilon_{j}+\sum_{i, j} \epsilon_{i} w_{i} \widehat{X}^{-1} \dot{X} \widehat{X}^{-1} \widehat{Y}^{-1} v_{j} \epsilon_{j}
$$

Now a quick look at (4.29), (4.18), (4.22) and (4.23) confirms that $\dot{M}=-\left(L^{k}\right)_{-} M$, as needed.

With flow (4.18) and (4.19) on $\mathcal{C}_{\alpha, \lambda}$ corresponding to the $k$ th flow of the hierarchy, we obtain a commuting family of Hamiltonian flows on $\mathcal{C}_{\alpha, \lambda}$ labelled by $k$. In fact, one can confirm by a direct calculation that the Hamiltonians (4.24) commute, that is,

$$
\left\{H_{j}, H_{k}\right\}=0 \quad \text { for all } j, k .
$$

Thus, we arrive at the following result.

Theorem 4.4. Under the map $\mathcal{C}_{\alpha, \lambda} \rightarrow \mathcal{P}$ which sends $(X, Y, v, w)$ to $L=M y M^{-1}$, the commuting flows with the Hamiltonians $H_{k}, k=1,2, \ldots$, get identified with the flows of the hierarchy (2.18).

Let us integrate flows (4.18) and (4.19) simultaneously for all $k$. First we integrate (4.19) to find that

$$
v=e^{-\sum_{k} t_{k} Y^{k}} v(0), \quad w=w(0) e^{\sum_{k} t_{k} Y^{k}}, \quad v w=e^{-\sum_{k} t_{k} \mathrm{ad}_{Y^{k}}}(v(0) w(0)),
$$

where $Y=Y(0)$ is constant and we use the standard notation for $a d_{A} B=A B-B A$. Next, note that (4.18) can be written as

$$
\frac{\partial X}{\partial t_{k}}=-\sum_{i \in I} e_{i+1}\left(\sum_{a=0}^{k-1} Y^{a} v w Y^{k-1-a}\right) e_{i}=-\sum_{i \in I} e_{i+1}\left(\operatorname{ad}_{Y}\right)^{-1} \operatorname{ad}_{Y^{k}}(v w) e_{i} .
$$

Substituting the expression for $v w$ and integrating, we find that

$$
X=X(0)+\sum_{i \in I} e_{i+1}\left\{\left(\operatorname{ad}_{Y}\right)^{-1}\left(e^{-\sum_{k} t_{k} \operatorname{ad}_{Y^{k}}}-1\right)(v(0) w(0))\right\} e_{i} .
$$

Note that $\left(\operatorname{ad}_{Y}\right)^{-1}\left(e^{-\sum_{k} t_{k} \operatorname{ad}_{Y^{k}}}-1\right)$ is well defined: after expanding the exponential into a series, each term contains a well-defined factor $\left(\operatorname{ad}_{Y}\right)^{-1} \operatorname{ad}_{Y}{ }^{k}$.

Let us remark on the flows with $k=m j, j \in \mathbb{N}$. In this case

$$
H_{m j}=-w Y^{m j} v=-\sum_{i \in I} w_{i} Y^{m j} v_{i}=-\operatorname{tr}\left(Y^{m j} \sum_{i} v_{i} w_{i}\right) .
$$

On $\mathcal{C}_{\alpha, \lambda}$ (3.24) holds, so $H_{m j}$ can be replaced by

$$
\widetilde{H}_{m j}=\operatorname{tr}\left(Y^{m j}\left(X Y-Y X+\sum_{i \in I} \lambda_{i} e_{i}\right)\right)=\sum_{i \in I} \lambda_{i} \operatorname{tr}\left(e_{i} Y^{m j} e_{i}\right)=\frac{|\lambda|}{m} \operatorname{tr} Y^{m j},
$$

where $|\lambda|:=\sum_{i \in I} \lambda_{i}$. According to (2.5), $|\lambda|=-m \tau \neq 0$. Therefore, flow (4.18) and (4.19) can be replaced by

$$
\begin{aligned}
\dot{X} & =j|\lambda| Y^{m j-1}, & \dot{Y} & =0, \\
\dot{v} & =0, & \dot{w} & =0 .
\end{aligned}
$$

As a result, the $m j$-th flow is linear: $X\left(t_{m j}\right)=X(0)+j|\lambda| t_{m j} Y^{m j-1}$. 


\section{Solutions for the spherical sub-hierarchy}

Given $\ell \in I$, consider quiver (3.15) and let $\mathcal{C}_{\alpha, \lambda}$ be a Calogero-Moser space (3.17). Recall that the description of $\mathcal{C}_{\alpha, \lambda}$ is almost the same as for quiver (3.14), see (3.28)-(3.30), the only difference being that $v_{i}=w_{i}=0$ for $i \neq \ell$. Therefore, in this case $v=v_{\ell}, w=w_{\ell}$ and we simply need to make appropriate changes in the previous formulas.

Hence, given a point $(X, Y, v, w)$ of $\mathcal{C}_{\alpha, \lambda}$, we put

$$
M=1-\epsilon_{\ell} w_{\ell}\left(X-x 1_{V}\right)^{-1}\left(Y-y 1_{V}\right)^{-1} v_{\ell} \epsilon_{\ell} .
$$

This defines a map $\mathcal{C}_{\alpha, \lambda} \rightarrow 1+\mathcal{P}_{-}$. By Lemma 4.1, the inverse of $M$ is given by

$$
M^{-1}=1+\epsilon_{\ell} w_{\ell}\left(Y-y 1_{V}\right)^{-1}\left(X-x 1_{V}\right)^{-1} v_{\ell} \epsilon_{\ell} .
$$

It is obvious from these formulas that $\epsilon_{i} M=M \epsilon_{i}$ and $\epsilon_{i} M^{-1}=M^{-1} \epsilon_{i}$ for $i \in I$. Hence, $L=M y M^{-1}$ and $\widetilde{L}=L^{m}=M y^{m} M^{-1}$ will satisfy

$$
\epsilon_{i} L=L \epsilon_{i-1}, \quad \epsilon_{i} \widetilde{L}=\widetilde{L} \epsilon_{i} .
$$

Now, using $(X, Y, v, w)$ as the initial data, we can construct a solution to the spherical sub-hierarchy. As explained above, when restricting to the flows with $k=m j$, the dependence of $(X, Y, v, w)$ on $t=\left(t_{m}, t_{2 m}, \ldots\right)$ reduces to

$$
X(t)=X(0)+|\lambda| \sum_{j \geqslant 1} j t_{m j} Y^{m j-1}, \quad Y=Y(0), \quad v=v(0), \quad w=w(0) .
$$

Clearly, the condition $v_{i}=w_{i}=0$ for $i \neq \ell$ remains valid for all times. As a result, the corresponding $L(t), \widetilde{L}(t)$ and $\widetilde{L}_{\ell}(t)=\epsilon_{\ell} \widetilde{L}(t)$ will satisfy Eqs. (2.24), (2.25), and (2.27).

Remark 4.5. Up to a change of notation, the element $M$ defined in (4.36) is the same as $\kappa$ in Ref. 26, Theorem 2.6; interpreting it as a "pseudo-differential operator" in a suitable sense was the starting point in our work.

Remark 4.6. The Calogero-Moser spaces (3.17) associated with quivers (3.14) and (3.15) are isomorphic to particular cases of the varieties from Refs. 4 and 5. Namely, they correspond to setting the $\Gamma$-module $W$ in Ref. 5, (2.16) as $W=\mathbb{C} \Gamma$ and $W=\mathbb{C} \epsilon_{\ell}$, respectively, where $\mathbb{C} \epsilon_{\ell}$ is viewed as a one-dimensional $\Gamma$-module. Similarly, the Calogero-Moser spaces for quivers (3.12) and (3.13) correspond to $W=\mathbb{C} \Gamma^{\oplus d}$ and $W=\left(\mathbb{C} \epsilon_{\ell}\right)^{\oplus d}$.

Remark 4.7. Above we assumed that $\lambda$ is regular, which puts restrictions (3.19) on parameters $k_{0}, \ldots, k_{m-1}$ for the Cherednik algebra $H_{\tau, k}$. Let us remark on the situation in general, for arbitrary $k_{i}$ and $\tau \neq 0$. In this case, one may have reducible representations in $\operatorname{Rep}\left(\Pi^{\lambda}, \boldsymbol{\alpha}\right)$. Non semi-simple representations are irrelevant because they are indistinguishable from semi-simple ones under the map $(X, Y, v, w) \mapsto M$. On the other hand, if $(X, Y, v, w)$ describes a semi-simple reducible $\Pi^{\lambda}$-module, then it is a direct sum of simples, exactly one of which must contain $V_{\infty}=\mathbb{C}$. Formula (4.1) makes it clear that $M$ is fully determined by this simple summand. Let $\alpha^{\prime}=\left(1, \alpha^{\prime}\right)$ be the dimension vector of this summand, so it corresponds to some point $\left(X^{\prime}, Y^{\prime}, v^{\prime}, w^{\prime}\right) \in \operatorname{Rep}\left(\Pi^{\lambda}, \boldsymbol{\alpha}^{\prime}\right)$. Then the two solutions of the hierarchy built from $(X, Y, v, w)$ and $\left(X^{\prime}, Y^{\prime}, v^{\prime}, w^{\prime}\right)$ coincide. Therefore, the solutions of the hierarchy are parametrised by the locus of simple representations

$$
\bigsqcup_{\alpha=(1, \alpha)} \operatorname{Irr}\left(\Pi^{\lambda}, \boldsymbol{\alpha}\right) / / G L(\alpha) .
$$

For a description of the set $\Sigma_{\lambda} \subset \Delta_{+}(Q)$ of all $\alpha$ such that $\operatorname{Irr}\left(\Pi^{\lambda}, \alpha\right) \neq \emptyset$, see Theorem 1.2 of Ref. 18.

Remark 4.8. The family of commuting Hamiltonians (4.24) can be generalised to any quiver $Q_{0}$ with a vertex set $I$. Namely, suppose $Q$ is obtained from $Q_{0}$ by adding a vertex $\infty$ and one arrow $v_{i}: \infty \rightarrow i$ for every $i \in I$. For $k \in \mathbb{N}$, let $h_{k}=\sum_{i, j \in I} v_{j}^{*} a_{k} \ldots a_{1} v_{i} \in \mathbb{C}[\bar{Q}]$, where the sum is taken over all paths $a_{k} \ldots a_{1}$ length $k$ in $Q_{0}$. It is a pleasant exercise to check that $\left[h_{k}, h_{l}\right]_{N L}=0$, 
where $[\cdot, \cdot]_{N L}$ denotes the necklace Lie bracket. ${ }^{14}$ According to the general principle, ${ }^{20,49}$ the functions $H_{k}=\operatorname{tr}\left(h_{k}\right)$ Poisson-commute both on $\operatorname{Rep}(\mathbb{C} \bar{Q}, \alpha)$ and on the symplectic quotients $\operatorname{Rep}\left(\Pi^{\lambda}, \alpha\right) / / G(\boldsymbol{\alpha})$.

\section{LINK TO INTEGRABLE PARTICLE DYNAMICS}

In this section, we explain how the dynamics (4.18) and (4.19) is related to the classical CalogeroMoser system for the wreath-product $\mathbb{Z}_{m}<S_{n}$. For this purpose, we will make the special choice $\alpha=(1, n \delta)$ where $\delta=(1, \ldots, 1)$. Consider the varieties $\mathcal{C}_{n \delta, \lambda}$ which will be abbreviated to $\mathcal{C}_{n, \lambda}$ for simplicity. Everything still depends on the choice of framing, so we have two versions of $\mathcal{C}_{n, \lambda}$, corresponding to quivers (3.14) and (3.15). We will deal first with the case (3.15).

\section{A. Darboux coordinates on $\mathcal{C}_{n, \lambda}$}

Consider quiver (3.15), where we set $\ell=0$ for simplicity. From formula (3.31), we find that $\operatorname{dim} \mathcal{C}_{n, \lambda}=2 n$. Points of $\mathcal{C}_{n, \lambda}$ are represented by linear maps

$$
X_{i}: V_{i} \rightarrow V_{i+1}, \quad Y_{i}: V_{i+1} \rightarrow V_{i}, \quad v_{0}: \mathbb{C} \rightarrow V_{0}, \quad w_{0}: V_{0} \rightarrow \mathbb{C},
$$

where $\operatorname{dim} V_{i}=n$. These maps should satisfy relation (3.28)

$$
Y_{i} X_{i}-X_{i-1} Y_{i-1}-\delta_{i 0} v_{0} w_{0}=\lambda_{i} 1_{V_{i}}, \quad(i \in \mathbb{Z} / m \mathbb{Z}) .
$$

To construct local coordinates on $\mathcal{C}_{n, \lambda}$, we look at the subset $\mathcal{C}_{n, \lambda}^{\prime} \subset \mathcal{C}_{n, \lambda}$ where the transformation $X_{m-1} \ldots X_{1} X_{0} \in \operatorname{End}\left(V_{0}\right)$ is diagonalisable and invertible. Suppose that this transformation has eigenvalues $x_{1}^{m}, \ldots, x_{n}^{m}$, then one can choose bases in each of $V_{i}$ so that all $X_{i}$ look identical

$$
X_{i}=\operatorname{diag}\left(x_{1}, \ldots, x_{n}\right)
$$

Then from (5.1), we obtain that

$$
\begin{aligned}
\left(Y_{i}\right)_{j j} x_{j}-x_{j}\left(Y_{i-1}\right)_{j j}-\delta_{i 0}\left(v_{0} w_{0}\right)_{j j} & =\lambda_{i}, \\
\left(Y_{i}\right)_{j k} x_{k}-x_{j}\left(Y_{i-1}\right)_{j k}-\delta_{i 0}\left(v_{0} w_{0}\right)_{j k} & =0 \quad(j \neq k) .
\end{aligned}
$$

Summing (5.3) over $i$, we find that for any $j$,

$$
\left(v_{0} w_{0}\right)_{j j}=-|\lambda|, \quad \text { where } \quad|\lambda|=\sum_{i \in I} \lambda_{i} \neq 0 .
$$

Therefore, by rescaling the bases in each of $V_{i}$, we may assume that

$$
\left(v_{0}\right)_{j}=1, \quad\left(w_{0}\right)_{j}=-|\lambda|, \quad \text { and therefore } \quad\left(v_{0} w_{0}\right)_{j k}=-|\lambda| \text { for all } j, k .
$$

The matrices $Y_{i}$ are then easily determined from relations (5.3) and (5.4). Namely, we must have $x_{j}^{m} \neq x_{k}^{m}$ for $j \neq k$, and the matrices $Y_{i}$ may be chosen in the following form:

$$
\left(Y_{i}\right)_{j j}=p_{j}+\frac{1}{x_{j}} \sum_{r=1}^{i} \lambda_{r}, \quad\left(Y_{i}\right)_{j k}=|\lambda| \frac{x_{j}^{i} x_{k}^{m-i-1}}{x_{j}^{m}-x_{k}^{m}} \quad(j \neq k),
$$

where $i=0,1, \ldots, m-1$.

We, therefore, can view $x=\left(x_{1}, \ldots, x_{n}\right)$ and $p=\left(p_{1}, \ldots, p_{n}\right)$ as local coordinates on $\mathcal{C}_{n, \lambda}^{\prime}$. Note that the set of eigenvalues $\left\{x_{1}^{m}, \ldots, x_{n}^{m}\right\}$ determines $x_{1}, \ldots, x_{n}$ only up to permutations and multiplication by the $m$ th roots of unity. As a result, the coordinates $\left\{x_{i}, p_{i}\right\}$ on $\mathcal{C}_{n, \lambda}^{\prime}$ are defined up to an action of the generalised symmetric group $W=\mathbb{Z}_{m} \imath S_{n}$. As a subgroup of $\mathrm{GL}(n, \mathbb{C}), W$ is generated by the premutation matrices $\sigma \in S_{n}$ and by transformations $s:\left(x_{1}, \ldots, x_{n}\right) \mapsto\left(\mu_{1} x_{1}, \mu_{2} x_{2}, \ldots, \mu_{n} x_{n}\right)$ with $\mu_{i}^{m}=1$. Embed $W$ into $\mathrm{GL}(\alpha)=\mathrm{GL}(n, \mathbb{C})^{m}$ in the following way:

$$
\sigma \mapsto(\sigma, \ldots, \sigma), \quad s \mapsto\left(1, s, \ldots, s^{m-1}\right) .
$$

When viewed as a subgroup of GL $(\alpha), W=\mathbb{Z}_{m}\left\langle S_{n}\right.$ acts on the representation space $\operatorname{Rep}\left(\left(\Pi^{\lambda}\right)^{\text {opp }}, \boldsymbol{\alpha}\right)$, thus on the above $X_{i}, Y_{i}, v_{0}, w_{0}$. It is easy to see that this corresponds to the "diagonal" action on $(x$, 
$p$ ): the action of $\sigma \in S_{n}$ permutes $\left\{x_{i}\right\}$ and $\left\{p_{i}\right\}$ simutaneously, while the action of $s$ changes $x_{i}, p_{i}$ to $\mu_{i} x_{i}, \mu_{i}^{-1} p_{i}$. If we regard $x, p$ as coordinates on $\mathfrak{h}:=\mathbb{C}^{n}$ and its dual, $\mathfrak{h}^{*}$, then $\mathcal{C}_{n, \lambda}^{\prime}$ becomes isomorphic to $\mathfrak{h}_{\text {reg }} \times \mathfrak{h}^{*} / W$, where $\mathfrak{h}_{\text {reg }}:=\left\{x \in \mathfrak{h} \mid x_{i}^{m} \neq x_{j}^{m} \neq 0\right.$ for all $\left.i \neq j\right\}$.

Recall that $\mathcal{C}_{n, \lambda}$ inherits a symplectic structure from the standard symplectic structure on the space $\operatorname{Rep}\left(\mathbb{C} \bar{Q}^{\text {opp }}, \boldsymbol{\alpha}\right)$,

$$
\omega=\sum_{i \in I} \operatorname{tr}\left(\mathrm{d} Y_{i} \wedge \mathrm{d} X_{i}\right)+\mathrm{d} w_{0} \wedge \mathrm{d} v_{0} .
$$

Denote this symplectic structure as $\omega_{\mathcal{C}_{n, \lambda}}$.

Proposition 5.1. The above coordinates $\left\{x_{i}, p_{i}\right\}$ are Darboux coordinates in the sense that

$$
\omega_{\mathcal{C}_{n, \lambda}}=m \sum_{i=1}^{n} \mathrm{~d} p_{i} \wedge \mathrm{d} x_{i}
$$

Proof. Recall that $\mathcal{C}_{n, \lambda}$ is obtained from $\operatorname{Rep}\left(\mathbb{C} Q^{\text {opp }}, \boldsymbol{\alpha}\right)$ by Hamiltonian reduction, see (3.6). Using the coordinates $\{x, p\}$, we identify $\mathcal{C}_{n, \lambda}^{\prime}$ locally with an open subset in $\mathfrak{h}_{\text {reg }} \times \mathfrak{h}^{*}$. Consider the mapping $\phi: \mathfrak{h}_{\text {reg }} \times \mathfrak{h}^{*} \rightarrow \operatorname{Rep}\left(\mathbb{C} \bar{Q}^{\text {opp }}, \boldsymbol{\alpha}\right)$, defined by formulas (5.2), (5.5), and (5.6). Locally, this represents a transversal slice for the action of $\operatorname{GL}(n, \mathbb{C})^{m}$ on $\operatorname{Rep}\left(\left(\Pi^{\lambda}\right)^{\text {opp }}, \boldsymbol{\alpha}\right) \subset \operatorname{Rep}\left(\mathbb{C} \bar{Q}^{\text {opp }}, \boldsymbol{\alpha}\right)$. Therefore, the symplectic structure on $\mathcal{C}_{n, \lambda}^{\prime}$ is the pullback $\phi^{*} \omega$ of the form (5.8). To calculate it, we substitute the expressions for $X_{i}, Y_{i}, v_{0}, w_{0}$ into (4.16). With $v_{0}$ and $w_{0}$ being constant and $\partial X_{i} / \partial p_{i}=0$, we get

$$
\phi^{*} \omega=\sum_{i \in I} \sum_{j<k} \operatorname{tr}\left(\frac{\partial Y_{i}}{\partial x_{j}} \frac{\partial X_{i}}{\partial x_{k}}-\frac{\partial Y_{i}}{\partial x_{k}} \frac{\partial X_{i}}{\partial x_{j}}\right) \mathrm{d} x_{j} \wedge \mathrm{d} x_{k}+\sum_{i \in I} \sum_{j, k} \operatorname{tr}\left(\frac{\partial Y_{i}}{\partial p_{j}} \frac{\partial X_{i}}{\partial x_{k}}\right) \mathrm{d} p_{j} \wedge \mathrm{d} x_{k} .
$$

Since each $X_{i}$ is a diagonal matrix, the off-diagonal part of $Y_{i}$ does not play any role and can be ignored. Then it is easy to see that the only non-zero terms are those with $j=k$, where we have $\operatorname{tr}\left(\frac{\partial Y_{i}}{\partial p_{j}} \frac{\partial X_{i}}{\partial x_{j}}\right)=1$. This leads to the result of the proposition.

Corollary 5.2. Let $Y=Y_{0}+\cdots+Y_{m-1}$, viewed as a linear endomorphism of $\mathbb{C}^{m n}=\oplus_{i} V_{i}$, with $Y_{i}: V_{i+1} \rightarrow V_{i}$ given by (5.6). Then $H_{j}=\frac{1}{m} \operatorname{tr}\left(Y^{j m}\right), j \in \mathbb{N}$ are Poisson-commuting functions on $\mathfrak{h}_{\text {reg }} \times \mathfrak{h}^{*}$.

\section{B. Relation to the Cherednik algebra and Calogero-Moser system}

For any finite complex reflection group $W \subset \mathrm{GL}(\mathfrak{h})$, Etingof and Ginzburg ${ }^{27}$ define the Cherednik algebra $H_{t, c}=H_{t, c}(W)$, depending on parameters $t, c$. For $t=0$, the algebra $H_{0, c}$ has a large center, denoted $Z_{0, c}$. The Calogero-Moser space associated with $W$ and $c$ is defined as $\operatorname{Spec} Z_{0, c}$ (Ref. 27, Definition 4.14); this is an algebraic Poisson variety.

Let $W=\mathbb{Z}_{m}<S_{n}$ act on $\mathfrak{h}=\mathbb{C}^{n}$ as before. This is the group $G(m, 1, n)$ in Shephard-Todd's classification of complex reflection groups ${ }^{48}\left[G(2,1, n)\right.$ is the Coxeter group of type $\left.B_{n}\right]$. The Cherednik algebra $H_{t, c}$ in this case depends on parameters $t \in \mathbb{C}$ and $c \in \mathbb{C}^{m}$. Recall that above we considered the varieties $\mathcal{C}_{n, \lambda}$ depending on $\lambda \in \mathbb{C}^{m}$.

Theorem 5.3 (Ref. 27, Theorem 1.13). Let $H_{t, c}$ be the Cherednik algebra of $W=\mathbb{Z}_{m} 2 S_{n}$. For generic $c \in \mathbb{C}^{m}$ and suitable $\lambda=\lambda(c)$, the algebraic Poisson varieties $\operatorname{Spec}\left(Z_{0, c}\right)$ and $\mathcal{C}_{n, \lambda}$ are isomorphic.

Remark 5.4. According to Ref. 41, Theorem 7.4, the genericity assumption on the parameters can be removed, i.e., the varieties $\mathcal{C}_{n, \lambda}$ and $\operatorname{Max} \operatorname{Spec}\left(Z_{0, c}\right)$ are isomorphic for any $c$ and $\lambda=\lambda(c)$.

Recall the construction of the Calogero-Moser Hamiltonians associated with $W=\mathbb{Z}_{m} \prec S_{n}$, see Refs. 33, 28, and 29. The Cherednik algebra $H_{1, c}$ has a commutative subalgebra that, in the Dunkl representation of $H_{1, c}$, is generated by $n$ Dunkl operators $T_{i}, i=1, \ldots, n$ (Ref. 25, Definition 3.2). These operators depend on parameters $\kappa=\left(\kappa_{0}, \kappa_{1}, \ldots, \kappa_{m-1}\right)$ and they act on polynomials $\mathbb{C}[\mathfrak{h}]$ as 
differential-reflection operators. The elements $T_{1}^{j m}+\cdots+T_{n}^{j m}, j \in \mathbb{N}$, when restricted on $W$-invariant polynomials, become commuting partial differential operators on $\mathfrak{h}$, which we denote as $L_{j}$. These are the quantum Hamiltonains of the Calogero-Moser system of type $\mathbb{Z}_{m}$ 々 $S_{n}$. A similar procedure exists for constructing their classical counterparts, which are Poisson-commuting functions $L_{j}(x, p)$ $\in \mathbb{C}\left[\mathfrak{b}_{\text {reg }} \times \mathfrak{h}^{*}\right]$, see Refs. 28 and 29. We have the following.

Proposition 5.5. Let $H_{j}=\frac{1}{m} \operatorname{tr}\left(Y^{j m}\right)$ be the Poisson-commuting functions as in Corollary 5.2. Let $L_{j}$ be the classical Hamiltonians of the Calogero-Moser system for $W=\mathbb{Z}_{m} 2 S_{n}$. Then, we have $H_{j}(x, p)=L_{j}(x, p)$ for certain $\kappa=\kappa(\lambda)$. Therefore, the competely integrable Hamiltonian system given by $H_{j}$ on the space $\mathcal{C}_{n, \lambda}$ in $x, p$ coordinates is equivalent to the Calogero-Moser system for $\mathbb{Z}_{m} \prec S_{n}$.

By Theorem 5.3, this result can be expected, so we only briefly indicate the steps in the proof. First, we recall that the isomorphism of Theorem 5.3 is constructed by associating with a point $z \in \operatorname{Spec}\left(Z_{0, c}\right)$ a pair of matrices $X, Y$. These $X, Y$ arise from the action of $x_{1}, T_{1} \in H_{0, c}$ on a certain invariant subspace $E^{\prime} \subset E$ of the representation of $H_{0, c}$, associated with $z$, see Ref. 27, Sec. 11. Next, on a dense open subset $U \subset \operatorname{Spec}\left(Z_{0, c}\right)$, the action of $x_{1}$ in a suitable basis of $E^{\prime}$ is diagonal. By the same method as in Ref. 28, Sec. 10.7, one then calculates the action of $T_{1}$ in this basis, using the explicit form of $T_{i}$ (Ref. 25, Definition 3.2). This gives an explicit formula for $Y$ and one observes directly that this coincides with $Y$ calculated in (5.6). Finally, one needs to check that $\operatorname{tr}\left(Y^{j m}\right)$ coincides with the (classical version of the) Hamiltonian $T_{1}^{j m}+\cdots+T_{n}^{j m}$. This is derived in the same way as Ref. 27, (11.30) and (11.31).

It follows that the matrix $Y$ is a Lax matrix for the Calogero-Moser system for $W=\mathbb{Z}_{m} \prec S_{n}$, in the sense that it deforms isospectrally under any of the commuting flows defined by the Hamiltonians $L_{j}(x, p)$, and the traces of powers of $Y$ produce a full set of first integrals in involution. The symplectic quotient $\mathcal{C}_{n, \lambda}$ may, therefore, be regarded as a completed phase space for this Calogero-Moser system.

Example 5.6. Consider the case $m=2$. In this case, $\lambda=\left(\lambda_{0}, \lambda_{1}\right)$, and $Y$ is a $2 n \times 2 n$ matrix of the form $\left(\begin{array}{rr}0 & Y_{1} \\ Y_{0} & 0\end{array}\right)$, with

$$
\begin{aligned}
& \left(Y_{0}\right)_{i i}=p_{i}, \quad\left(Y_{0}\right)_{i j}=|\lambda| \frac{x_{j}}{x_{i}^{2}-x_{j}^{2}}, \\
& \left(Y_{1}\right)_{i i}=p_{i}+\frac{\lambda_{1}}{x_{i}}, \quad\left(Y_{1}\right)_{i j}=|\lambda| \frac{x_{i}}{x_{i}^{2}-x_{j}^{2}} .
\end{aligned}
$$

It is equivalent to the well-known Lax matrix for the rational Calogero-Moser system in type $B_{n},{ }^{44}$ cf. Ref. 9, Eq. (4.48). We have

$$
\frac{1}{2} \operatorname{tr} Y^{2}=\sum_{i=1}^{n} p_{i}\left(p_{i}+\frac{\lambda_{1}}{x_{i}}\right)-\sum_{i<j} \frac{|\lambda|^{2}\left(x_{i}^{2}+x_{j}^{2}\right)}{\left(x_{i}^{2}-x_{j}^{2}\right)^{2}} .
$$

After a canonical transformation $p_{i} \mapsto p_{i}-\frac{\lambda_{1}}{2 x_{i}}$ this takes the conventional form

$$
\sum_{i=1}^{n}\left(p_{i}^{2}-\frac{\lambda_{1}^{2}}{4 x_{i}^{2}}\right)-\sum_{i<j} \frac{|\lambda|^{2}\left(x_{i}^{2}+x_{j}^{2}\right)}{\left(x_{i}^{2}-x_{j}^{2}\right)^{2}} .
$$

Note that setting $\lambda_{1}=0$ leads to the $D_{n}$-case.

\section{Spin particle dynamics}

For the full hierarchy (2.18), the solutions are parameterised by the varieties (3.17) for the quiver $Q$ as in (3.14). As in Sec. V A, let us consider the special choice of the dimension vector $\alpha=(1, n \delta)$ 
where $\delta=(1, \ldots, 1)$. Let us again abbreviate the varieties $\mathcal{C}_{n \delta, \lambda}(Q)$ to $\mathcal{C}_{n, \lambda}$ for simplicity. From formula (3.27), we find that $\operatorname{dim} \mathcal{C}_{n, \lambda}=2 m n$. Points of $\mathcal{C}_{n, \lambda}$ are represented by linear maps

$$
X_{i}: V_{i} \rightarrow V_{i+1}, \quad Y_{i}: V_{i+1} \rightarrow V_{i}, \quad v_{i}: \mathbb{C} \rightarrow V_{i}, \quad w_{i}: V_{i} \rightarrow \mathbb{C},
$$

satisfying relations (3.21),

$$
Y_{i} X_{i}-X_{i-1} Y_{i-1}-v_{i} w_{i}=\lambda_{i} 1_{V_{i}} \quad(i \in I) .
$$

Similarly to the previous case, we look at the subset $\mathcal{C}_{n, \lambda}^{\prime} \subset \mathcal{C}_{n, \lambda}$ where $X_{m-1} \ldots X_{1} X_{0}$ has distinct non-zero eigenvalues $x_{1}^{m}, \ldots, x_{n}^{m}$. Then by choosing suitable bases in each of $V_{i}$, we will have

$$
X_{i}=\operatorname{diag}\left(x_{1}, \ldots, x_{n}\right)
$$

implying that

$$
\begin{aligned}
\left(Y_{i}\right)_{j j} x_{j}-x_{j}\left(Y_{i-1}\right)_{j j}-\left(v_{i} w_{i}\right)_{j j} & =\lambda_{i}, \\
\left(Y_{i}\right)_{j k} x_{k}-x_{j}\left(Y_{i-1}\right)_{j k}-\left(v_{i} w_{i}\right)_{j k} & =0 \quad(j \neq k) .
\end{aligned}
$$

Summing (5.11) over $i$, we obtain that for any $j$,

$$
\sum_{i \in I}\left(v_{i} w_{i}\right)_{j j}=-|\lambda|
$$

The matrices $Y_{i}$ are then found to be as follows:

$$
\begin{aligned}
& \left(Y_{i}\right)_{j j}=p_{j}+\frac{1}{x_{j}} \sum_{r=0}^{i}\left(\lambda_{r}+\left(v_{r} w_{r}\right)_{j j}\right)+\frac{1}{x_{j}} \sum_{r=0}^{m-1} \frac{r-m}{m}\left(\lambda_{r}+\left(v_{r} w_{r}\right)_{j j}\right), \\
& \left(Y_{i}\right)_{j k}=-\sum_{l=0}^{m-1} \frac{\left(v_{i-l} w_{i-l}\right)_{j k} x_{j}^{l} x_{k}^{m-l-1}}{x_{j}^{m}-x_{k}^{m}} \quad(j \neq k),
\end{aligned}
$$

where $i=0,1, \ldots, m-1$ (the index $i-l$ in the second formula is taken modulo $m$ ).

Remark 5.7. The second sum in the right hand side of (5.13) is added to have the relation $\sum_{i \in I}\left(Y_{i}\right)_{j j}=m p_{j}$. This choice is important for Proposition 5.8 below.

It seems convenient to organise the column and row vectors $v_{i}, w_{i}$ into matrices $v$ and $w$ of size $n \times m$ and $m \times n$, respectively, and then divide them into rows and columns, each of length $m$. This way we get $n$ rows $\varphi_{j}$ and $n$ columns $\psi_{j}$, with $\left(v_{i}\right)_{j}=\left(\varphi_{j}\right)_{i}$ and $\left(w_{i}\right)_{j}=\left(\psi_{j}\right)_{i}$. The formulas for $Y_{i}$ then become as follows:

$$
\begin{aligned}
& \left(Y_{i}\right)_{j j}=p_{j}+\frac{1}{x_{j}} \sum_{r=0}^{i}\left(\lambda_{r}+\left(\psi_{j} \varphi_{j}\right)_{r r}\right)+\frac{1}{x_{j}} \sum_{r=0}^{m-1} \frac{r-m}{m}\left(\lambda_{r}+\left(\psi_{j} \varphi_{j}\right)_{r r}\right), \\
& \left(Y_{i}\right)_{j k}=-\sum_{l=0}^{m-1} \frac{\left(\psi_{k} \varphi_{j}\right)_{i-l, i-l} x_{j}^{l} x_{k}^{m-l-1}}{x_{j}^{m}-x_{k}^{m}} \quad(j \neq k),
\end{aligned}
$$

with the constraint $\varphi_{j} \psi_{j}=-|\lambda|$ for all $j$. Note that acting on each $V_{i}$ by a diagonal matrix with diagonal entries $d_{1}, \ldots, d_{n}$, in accordance with (3.26), leaves $x_{j}, p_{j}$ unchanged and replaces $\varphi_{j}$ and $\psi_{j}$ by $d_{j} \varphi_{j}$ and $d_{j}^{-1} \psi_{j}$. By analogy with Ref. 32 (cf. Ref. 52), the quantities $\varphi_{j}, \psi_{j}$ can be thought of as spin variables attached to the $j$ th particle of an $n$-particle system of the Calogero-Moser type. With each pair $\left(\varphi_{j}, \psi_{j}\right)$, we can therefore associate a point $q_{j}$ of the symplectic variety $Q_{m}$ (cf. Ref. 52)

$$
Q_{m}=\left\{(\varphi, \psi) \in\left(\mathbb{C}^{m}\right)^{*} \oplus \mathbb{C}^{m}|\varphi \psi=-| \lambda \mid\right\} / \mathbb{C}^{\times} .
$$

The above formulas then give a local parameterisation of $\mathcal{C}_{n, \lambda}^{\prime}$ by $\left\{x_{j}, p_{j}, q_{j}\right\}$.

By an elementary residue calculation,

$$
\frac{x_{j}^{l} x_{k}^{m-l-1}}{x_{j}^{m}-x_{k}^{m}}=\frac{1}{m} \sum_{r=0}^{m-1} \frac{\mu^{-l r}}{\mu^{r} x_{j}-x_{k}} .
$$


As a result, formula (5.16) can be replaced with

$$
\left(Y_{i}\right)_{j k}=\sum_{r \in I} \frac{\mu^{-i r}\left(\varphi_{j} E^{r} \psi_{k}\right)}{m\left(x_{k}-\mu^{r} x_{j}\right)}, \quad E^{r}=\operatorname{diag}\left(1, \mu^{r}, \mu^{2 r}, \ldots, \mu^{(m-1) r}\right) .
$$

Recall that the symplectic structure (4.16) induces a symplectic structure on $\mathcal{C}_{n, \lambda}$.

Proposition 5.8. In terms of $x_{j}, p_{j}, q_{j}=\left(\varphi_{j}, \psi_{j}\right)$, the symplectic structure on $\mathcal{C}_{n, \lambda}$ has the form

$$
\omega_{\mathcal{C}_{n, \lambda}}=\sum_{j=1}^{n}\left(m \mathrm{~d} p_{j} \wedge \mathrm{d} x_{j}-\mathrm{d} \varphi_{j} \wedge \mathrm{d} \psi_{j}\right) .
$$

Here, by abuse of notation, we do not make distinction between the form $\mathrm{d} \varphi_{j} \wedge \mathrm{d} \psi_{j}=\sum_{r=0}^{m-1} \mathrm{~d} \varphi_{j, r} \wedge \mathrm{d} \psi_{j, r}$ on $T^{*} \mathbb{C}^{m}$ and the corresponding symplectic form on $Q_{m}$.

Proof. The symplectic form on $\operatorname{Rep}\left(\mathbb{C} \bar{Q}^{\text {opp }}, \boldsymbol{\alpha}\right)$ in this case is

$$
\omega=\sum_{i \in I}\left(\operatorname{tr}\left(\mathrm{d} Y_{i} \wedge \mathrm{d} X_{i}\right)+\mathrm{d} w_{i} \wedge \mathrm{d} v_{i}\right) .
$$

Let us view formulas (5.10), (5.15), and (5.16) as describing a map

$$
\phi: T^{*} \mathfrak{b}_{\mathrm{reg}} \times\left(T^{*} \mathbb{C}^{m}\right)^{n} \rightarrow \operatorname{Rep}\left(\mathbb{C} \bar{Q}^{\text {opp }}, \boldsymbol{\alpha}\right) .
$$

Similarly to the proof of Proposition 5.1, we need to calculate $\phi^{*} \omega$. In the same way, only the diagonal entries of $Y_{i}$ will contribute to $\phi^{*} \omega$. We have

$$
\mathrm{d}\left(Y_{i}\right)_{j j}=\mathrm{d} p_{j}+\frac{1}{x_{j}}\left(\sum_{r=0}^{i} \mathrm{~d}\left(\psi_{j} \varphi_{j}\right)_{r r}+\sum_{r=0}^{m-1} \frac{r-m}{m} \mathrm{~d}\left(\psi_{j} \varphi_{j}\right)_{r r}\right)+\frac{\partial\left(Y_{i}\right)_{j j}}{\partial x_{j}} \mathrm{~d} x_{j} .
$$

The last term will vanish after taking the wedge product with $\mathrm{d}\left(X_{i}\right)_{j j}=\mathrm{d} x_{j}$. A simple calculation then shows that $\sum_{i=0}^{m-1} \mathrm{~d}\left(Y_{i}\right)_{j j} \wedge \mathrm{d}\left(X_{i}\right)_{j j}=m \mathrm{~d} p_{j} \wedge \mathrm{d} x_{j}$.

The Hamiltonians $H_{k}$ (4.24) after substituting (5.15) and (5.16) produce Poisson commuting functions on the phase space $\mathfrak{h}_{\text {reg }} \times \mathfrak{h}^{*} \times\left(Q_{m}\right)^{n}$ equipped with the symplectic form $\omega_{\mathcal{C}_{n, \lambda}}$. They define a system which can be interpreted as a spin version of the $n$-particle Calogero-Moser system for $W=\mathbb{Z}_{m} \prec S_{n}$, with the spin variables $\left(\varphi_{j}, \psi_{j}\right) \in Q_{m}$. It is a special case of a more general system with spin variables, see Subsection VI C.

Note that the choice of coordinates on $\mathcal{C}_{n, \lambda}^{\prime}$ is only unique up to the action of $G$, coming from embedding (5.7). Explicitly in coordinates this action permutes $\left\{x_{j}\right\},\left\{p_{j}\right\},\left\{q_{j}=\left(\varphi_{j}, \psi_{j}\right)\right\}$ simultaneously and replaces $x_{j}, p_{j}, \varphi_{j}$, and $\psi_{j}$ by $x^{r_{j}} x_{j}, \mu^{-r_{j}} p_{j}, \varphi_{j} E^{r_{j}}$, and $E^{-r_{j}} \psi_{j}$, respectively, where $E^{r}$ is the same as in (5.17). It follows that $\mathcal{C}_{n, \lambda}^{\prime} \simeq \mathfrak{h}_{\text {reg }} \times \mathfrak{h}^{*} \times\left(Q_{m}\right)^{n} / W$. We obtain the following result.

Proposition 5.9. Let $H_{k}=H_{k}(x, p, q)$ be the functions of $(x, p, q) \in \mathfrak{h}_{\text {reg }} \times \mathfrak{h}^{*} \times\left(Q_{m}\right)^{n}$, obtained by substituting (5.15) and (5.16) into (4.24). Then $H_{k}$ with $k=1, \ldots$, mn are functionally independent, Poisson commuting functions and hence define a completely integrable system. The Hamiltonian flows defined by $H_{k}$ extend from $\mathfrak{h}_{\mathrm{reg}} \times \mathfrak{h}^{*} \times\left(Q_{m}\right)^{n} / W$ to produce complete flows on the Calogero-Moser space $\mathcal{C}_{n, \lambda}=\mathcal{C}_{n \delta, \lambda}$ for quiver (3.14).

The number of integrals, $m n$, is clearly the half of the dimension of the phase space $\mathfrak{h}_{\text {reg }} \times \mathfrak{h}^{*} \times\left(Q_{m}\right)^{n}$, so the only thing to prove is that $H_{k}$ with $k=1, \ldots, m n$ is independent. This is shown in a more general case in Sec. VI (Proposition 6.7).

Example 5.10. Consider the case $m=2$. Let us write down the first two Hamiltonians in the coordinates $x_{j}, p_{j}, q_{j}$. In this case, we have two-component spin variables $\varphi_{j}=\left(\varphi_{j 0}, \varphi_{j 1}\right)$ and 


$$
\begin{aligned}
& \psi_{j}=\left(\psi_{j 0}, \psi_{j 1}\right)^{\top} \text {. Denote } E=\left(\begin{array}{rr}
1 & 0 \\
0 & -1
\end{array}\right) \text { and } F_{ \pm}=\left(\begin{array}{rr}
0 & \pm 1 \\
1 & 0
\end{array}\right) . \text { Then we have } \\
& H_{1}=-\sum_{i=0,1} w_{i} Y_{i} v_{i+1}=-\sum_{j=1}^{n}\left(\left(\varphi_{j} F_{+} \psi_{j}\right) p_{j}+\frac{1}{2 x_{j}}\left(\varphi_{j} F_{-} \psi_{j}\right)\left(\lambda_{0}+\varphi_{j 0} \psi_{j 0}\right)\right) \\
& -\sum_{j \neq k}\left(\frac{\left(\varphi_{j} F_{+} \psi_{k}\right)\left(\varphi_{k} \psi_{j}\right)}{2\left(x_{j}-x_{k}\right)}+\frac{\left(\varphi_{j} F_{-} \psi_{k}\right)\left(\varphi_{k} E \psi_{j}\right)}{2\left(x_{j}+x_{k}\right)}\right), \\
& H_{2}=\frac{|\lambda|}{2} \operatorname{tr} Y^{2}=|\lambda| \sum_{j=1}^{n}\left(p_{j}^{2}-\frac{1}{4 x_{j}^{2}}\left(\lambda_{0}+\varphi_{j 0} \psi_{j 0}\right)^{2}\right) \\
& +|\lambda| \sum_{j \neq k}\left(\frac{\left(\varphi_{j} \psi_{k}\right)\left(\varphi_{k} \psi_{j}\right)}{4\left(x_{j}-x_{k}\right)^{2}}+\frac{\left(\varphi_{j} E \psi_{k}\right)\left(\varphi_{k} E \psi_{j}\right)}{4\left(x_{j}+x_{k}\right)^{2}}+\frac{\varphi_{j 1} \psi_{k 1} \varphi_{k 0} \psi_{j 0}-\varphi_{j 0} \psi_{k 0} \varphi_{k 1} \psi_{j 1}}{2\left(x_{j}^{2}-x_{k}^{2}\right)}\right),
\end{aligned}
$$

where $\left(\lambda_{0}+\varphi_{j 0} \psi_{j 0}\right)=-\left(\lambda_{1}+\varphi_{j 1} \psi_{j 1}\right)$.

In the case $m=2$, when $W$ is of $B_{n}$-type, some spin versions of Calogero-Moser problem can be constructed in the framework of Lie algebras, see Ref. 39 and references therein. However they look somewhat different to our system. To the best of our knowledge, for $m>2$ spin generalizations of the Calogero-Moser system for $W=\mathbb{Z}_{m} \prec S_{n}$ have not been considered in the literature.

\section{MULTICOMPONENT GENERALISATION}

The KP hierarchy admits a multicomponent (matrix) generalisation, ${ }^{22,47}$ see also Ref. 23 . In the same spirit, one can generalise the hierarchy (2.18). Below we introduce such a hierarchy and construct its solutions by using a quiver

\section{A. Definition of the hierarchy}

We will follow closely the notation of Ref. 23 .

We will work with the ring $\operatorname{Mat}(d, \mathbb{C}) \otimes \mathcal{P}$ of matrices over the ring $\mathcal{P}(2.13)$. The operations $(\cdot)_{+}$ and $(\cdot)_{-}$on $\mathcal{P}$ induce similar operations on this ring.

Let $E_{\alpha}(\alpha=1, \ldots, d)$ be the diagonal matrices with the diagonal entries $\left(E_{\alpha}\right)_{\beta \beta}=\delta_{\alpha \beta}$. We have $E_{\alpha} E_{\beta}=\delta_{\alpha \beta} E_{\alpha}$ and $E_{1}+\cdots+E_{d}=1$.

Now consider the elements of $\operatorname{Mat}(d, \mathbb{C}) \otimes \mathcal{P}$ of the following form:

$$
\begin{aligned}
L & =y+\sum_{l=0}^{\infty} f_{l} y^{-l}, \\
R_{\alpha} & =E_{\alpha}+\sum_{l=1}^{\infty} r_{l \alpha} y^{-l} \quad(\alpha=1, \ldots, d),
\end{aligned}
$$

where $f_{l}, r_{l \alpha} \in \operatorname{Mat}(d, \mathbb{C}) \otimes(\mathbb{C}(x) * \Gamma)$.

We impose the following relations on $L$ and $R_{\alpha}$,

$$
\left[L, R_{\alpha}\right]=0, \quad R_{\alpha} R_{\beta}=\delta_{\alpha \beta} R_{\alpha}, \quad \sum_{\alpha=1}^{d} R_{\alpha}=1 .
$$

The matrix generalisation of the hierarchy (2.18) is the following system of equations on $\left\{L, R_{\alpha}\right\}$ :

$$
\begin{aligned}
& \frac{\partial L}{\partial t_{k \beta}}=\left[\left(L^{k} R_{\beta}\right)_{+}, L\right], \\
& \frac{\partial R_{\alpha}}{\partial t_{k \beta}}=\left[\left(L^{k} R_{\beta}\right)_{+}, R_{\alpha}\right],
\end{aligned}
$$

where $t_{k \beta}(\beta=1, \ldots, d, k=0,1, \ldots)$ are the time variables. Note that it follows from (6.3) that $\sum_{\beta=1}^{d} \frac{\partial}{\partial t_{0 \beta}}=0$. 
The following proposition and its proof are parallel to Proposition 2.4.

Proposition 6.1. (1) Equations (6.4) and (6.5) imply the zero-curvature equations

$$
\left[\frac{\partial}{\partial t_{k \beta}}-\left(L^{k} R_{\alpha}\right)_{+}, \frac{\partial}{\partial t_{l \beta}}-\left(L^{l} R_{\beta}\right)_{+}\right]=0 \quad(k, l \geqslant 0) .
$$

(2) Flows (6.4) and (6.5) pairwise commute and preserve relations (6.3).

We will also impose the following constraint analogous to (2.20):

$$
\left(f_{0, \alpha \alpha}\right)_{e}=0 \quad(\alpha=1, \ldots, d),
$$

where $f_{0, \alpha \alpha} \in \mathbb{C}(x) * \Gamma$ are the diagonal entries of the coefficient $f_{0}$ in (6.1). These conditions (6.7) are preserved by flows (6.4) and (6.5).

For $M \in 1+\operatorname{Mat}(d, \mathbb{C}) \otimes \mathcal{P}_{-}$, define $L$ and $R_{\alpha}$ by the following formulas:

$$
L=M y M^{-1}, \quad R_{\alpha}=M E_{\alpha} M^{-1} .
$$

It is clear that these elements have the form (6.1) and (6.2) and satisfy (6.3) and (6.7). Note that $L^{k} R_{\alpha}=M y^{k} E_{\alpha} M^{-1}$.

Proposition 6.2. (cf. Proposition 2.6). Suppose $M \in 1+\operatorname{Mat}(d, \mathbb{C}) \otimes \mathcal{P}_{-}$depends on $t_{k \beta}$ and satisfies the equation

$$
\frac{\partial M}{\partial t_{k \beta}}=-\left(M y^{k} E_{\beta} M^{-1}\right)_{-} \cdot M
$$

then $L$ and $R_{\alpha}$ defined by (6.8) satisfy Eqs. (6.4) and (6.5).

Remark 6.3. A more general hierarchy can be constructed by allowing in (6.3)-(6.5) $L$ of the form

$$
L=A y+\sum_{l=0}^{\infty} f_{l} y^{-l},
$$

where $A=\operatorname{diag}\left(a_{1}, \ldots, a_{d}\right)$ with non-zero $a_{\alpha} \in \mathbb{C}$ (cf. Ref. 23). Solutions to this hierarchy can then be constructed as follows. Let $M$ be an element satisfying the assumptions of Proposition 6.2. Then $L_{A}=M A y M^{-1}$ and $R_{\alpha}=M E_{\alpha} M^{-1}$ satisfy Eqs. (6.3)-(6.5) after rescaling the time variables by $t_{k \beta}$ $\rightarrow a_{\beta}^{-k} t_{k \beta}$.

\section{B. Constructions of solutions}

We are going to construct solutions of the hierarchy (6.3)-(6.5), parametrised by Calogero-Moser spaces associated with quivers (3.12) and (3.13).

Let $Q$ be quiver (3.12) and $\mathcal{C}_{\alpha, \lambda}=\mathcal{C}_{\alpha, \lambda}(Q)$ be the Calogero-Moser space (3.17)

$$
\mathcal{C}_{\alpha, \lambda}(Q)=\operatorname{Rep}\left(\left(\Pi^{\lambda}\right)^{\text {opp }}, \boldsymbol{\alpha}\right) / / \operatorname{GL}(\alpha) .
$$

Recall that here $\alpha \in \mathbb{N}_{0}^{m}, \lambda \in \mathbb{C}^{m}$ and $\alpha=(1, \alpha), \lambda=(-\lambda \cdot \alpha, \lambda)$. A point of $\mathcal{C}_{\alpha, \lambda}$ represented by a collection of maps

$$
\begin{aligned}
& X_{i}: V_{i} \rightarrow V_{i+1}, \quad Y_{i}: V_{i+1} \rightarrow V_{i}, \\
& v_{i, \alpha}: \mathbb{C} \rightarrow V_{i}, \quad w_{i, \alpha}: V_{i} \rightarrow \mathbb{C}, \quad(\alpha=1, \ldots, d),
\end{aligned}
$$

corresponding to arrows $a_{i}, a_{i}^{*}, b_{i, \alpha}$, and $b_{i, \alpha}^{*}$, respectively. These maps should satisfy the relations [cf. (3.21) and (3.22)].

$$
\begin{aligned}
Y_{i} X_{i}-X_{i-1} Y_{i-1}-\sum_{\alpha=1}^{d} v_{i, \alpha} w_{i, \alpha} & =\lambda_{i} 1_{V_{i}}, \\
\sum_{i \in I} \sum_{\alpha=1}^{d} w_{i, \alpha} v_{i, \alpha} & =\lambda_{\infty} .
\end{aligned}
$$

[As before, (6.13) follows from (6.12).] 
Let us define maps

$$
v_{i}: \mathbb{C}^{d} \rightarrow V_{i}, \quad w_{i}: V_{i} \rightarrow \mathbb{C}^{d},
$$

by $v_{i}=\left(v_{i, 1}, \ldots, v_{i, d}\right)$ and $w_{i}=\left(w_{i, 1}, \ldots, w_{i, d}\right)$. Then relations $(6.12)$ take the form

$$
Y_{i} X_{i}-X_{i-1} Y_{i-1}-v_{i} w_{i}=\lambda_{i} 1_{V_{i}},
$$

looking similar to (3.21).

Introducing $V=V_{0} \oplus \ldots \oplus V_{m-1}$ and $X, Y \in \operatorname{End}(V)$ as in (3.23), relation (3.24) still holds true.

We will be assuming that $\lambda$ is regular in the sense of (3.18). Then using Proposition 3.1 we find, similarly to (3.27), that

$$
\operatorname{dim} \mathcal{C}_{\alpha, \lambda}=2 \sum_{i \in I}\left(\alpha_{i} \alpha_{i+1}+d \alpha_{i}-\alpha_{i}^{2}\right)=2 d \sum_{i \in I} \alpha_{i}-\sum_{i \in I}\left(\alpha_{i}-\alpha_{i+1}\right)^{2} .
$$

Recall that the space $\mathcal{C}_{\alpha, \lambda}$ is a symplectic quotient [see (3.6)]. The symplectic structure on it is induced by the symplectic structure on $\operatorname{Rep}\left((\mathbb{C} Q)^{\text {opp }}, \boldsymbol{\alpha}\right)$ which has the form

$$
\omega=\sum_{i \in I}\left(\operatorname{tr}\left(\mathrm{d} Y_{i} \wedge \mathrm{d} X_{i}\right)+\operatorname{tr}\left(\mathrm{d} w_{i} \wedge \mathrm{d} v_{i}\right)\right)
$$

The Poisson brackets corresponding to (6.16) are

$$
\left\{\left(X_{i}\right)_{a b},\left(Y_{j}\right)_{c d}\right\}=\delta_{i j} \delta_{a d} \delta_{b c}, \quad\left\{\left(v_{i, \alpha}\right)_{a},\left(w_{j, \beta}\right)_{b}\right\}=\delta_{i j} \delta_{a b} \delta_{\alpha \beta} .
$$

Following the idea of Ref. 32, consider the following functions on $\mathcal{C}_{\alpha, \lambda}$ :

$$
I_{k}(A):=\sum_{i \in I} \operatorname{tr}\left(A w_{i} Y^{k} v_{i+k}\right), \quad A \in \operatorname{Mat}(d, \mathbb{C}) .
$$

The functions $I_{k}(A)$ with $k=0,1, \ldots$ and $A \in \operatorname{Mat}(d, \mathbb{C})$ form a vector space, closed under the Poisson bracket

$$
\left\{I_{k}(A), I_{l}(B)\right\}=I_{k+l}([B, A]) .
$$

Choosing $A=-E_{\beta}(\beta=1, \ldots, d)$, we get Poisson-commuting functions $H_{k, \beta}:=-I_{k}\left(E_{\beta}\right)$. The Hamiltonian flow on $\mathcal{C}_{\alpha, \lambda}$ looks as follows:

$$
\begin{array}{ll}
\dot{X}=-\sum_{l=0}^{m-1} \sum_{a=0}^{k-1} Y^{a} v_{l} E_{\beta} w_{l-k} Y^{k-a-1}, & \dot{Y}=0, \\
\dot{w}_{i}=E_{\beta} w_{i-k} Y^{k}, & \dot{v}_{j}=-Y^{k} v_{j+k} E_{\beta} .
\end{array}
$$

Note that these equations can be integrated explicitly similarly to (4.31) and (4.32).

To construct solutions of the hierarchy (6.4) and (6.5), let us introduce an element $M \in \operatorname{Mat}(d, \mathbb{C})$ $\otimes \mathcal{P}$ by the same formula (4.1)

$$
M=1-\sum_{i, j \in I} \epsilon_{i} w_{i}\left(X-x 1_{V}\right)^{-1}\left(Y-y 1_{V}\right)^{-1} v_{j} \epsilon_{j}
$$

Proposition 6.4. Let $L=M y M^{-1}$ and $R_{\alpha}=M E_{\alpha} M^{-1}$ with $M$ given by (6.20). If $X, Y, v_{i}, w_{i}$ satisfy Eqs. (6.18) and (6.19), then $L$ and $R_{\alpha}(\alpha=1, \ldots, d)$ satisfy the equations $\dot{L}=\left[\left(L^{k} R_{\beta}\right)_{+}, L\right]$ and $\dot{R}_{\alpha}=\left[\left(L^{k} R_{\beta}\right)_{+}, R_{\alpha}\right]$.

Proof. Proof essentially repeats the arguments from Sec. IV. 
First, since the relations for $X, Y, v_{i}, w_{i}$ have the same form, the identities (4.4)-(4.7) and Lemma 4.1 carry through. Furthermore, $L R_{\beta}=M y^{k} E_{\beta} M^{-1}$ and therefore $L R_{\beta}$ can be found from the right hand side of (4.11) by substituting $y^{k} \rightarrow y^{k} E_{\beta}$. After that, repeating the same steps as in the proof of Lemma 4.2, we obtain that

$$
\begin{aligned}
&\left(M y^{k} E_{\beta} M^{-1}\right)_{-}=\left(L^{k} R_{\beta}\right)_{-}=\sum_{i, j} \epsilon_{i} E_{\beta} w_{i-k} Y^{k} \widehat{Y}^{-1} \widehat{X}^{-1} v_{j} \epsilon_{j} \\
&-\sum_{i, j} \epsilon_{i} w_{i} \widehat{X}^{-1} \widehat{Y}^{-1} Y^{k} v_{j+k} E_{\beta} \epsilon_{j}-\sum_{i, j, \ell} \epsilon_{i} w_{i} \widehat{X}^{-1} \widehat{Y}^{-1} Y^{k} v_{\ell} E_{\beta} w_{\ell-k} \widehat{Y}^{-1} \widehat{X}^{-1} v_{j} \epsilon_{j} \\
&+\sum_{i, j, \ell} \sum_{a=0}^{k-1} \epsilon_{i} w_{i} \widehat{X}^{-1} Y^{a} v_{\ell} E_{\beta} w_{\ell-k} Y^{k-1-a} \widehat{Y}^{-1} \widehat{X}^{-1} v_{j} \epsilon_{j} .
\end{aligned}
$$

Note that the right-hand side of this expression is obtained from (4.10) by replacing

$$
w_{i-k} \rightarrow E_{\beta} w_{i-k}, \quad v_{j+k} \rightarrow v_{j+k} E_{\beta}, \quad v_{\ell} w_{\ell-k} \rightarrow v_{\ell} E_{\beta} w_{\ell-k} .
$$

It is then easy to check that the proof of Proposition 4.3 can be repeated by making the replacements (6.22) throughout.

Now varying $\beta$ and $k$ in (6.18) and (6.19), we have a family of commuting Hamiltonian flows on $\mathcal{C}_{\alpha, \lambda}$, so by the above Proposition, we obtain solutions of the hierarchy.

Theorem 6.5. The map $\mathcal{C}_{\alpha, \lambda} \rightarrow(\operatorname{Mat}(d, \mathbb{C}) \otimes \mathcal{P})^{d+1}$, which assigns to $\left(X, Y, v_{i}, w_{i}\right)$ the elements $L$ and $R_{\alpha}$ given by (6.8) and (6.20), sends the Hamiltonian flows (6.18) and (6.19) to the flows of the matrix hierarchy (6.4) and (6.5). Therefore solutions of the hierarchy can be found by integrating flows (6.18) and (6.19) with an arbitrary initial point in $\mathcal{C}_{\alpha, \lambda}$.

\section{Darboux coordinates and integrable dynamics}

For the full hierarchy, the solutions are parameterised by the varieties (3.17) for the quiver $Q$ as in (3.14). As in Sec. V C, let us consider the Calogero-Moser space $\mathcal{C}_{\alpha, \lambda}$ for the special choice of the dimension vector $\alpha=n \delta$ where $\delta=(1, \ldots, 1)$. Let $\mathcal{C}_{n, \lambda}$ denote this space; formula (6.15) gives us that $\operatorname{dim} \mathcal{C}_{n, \lambda}=2 m n d$. The construction of Darboux coordinates on $\mathcal{C}_{n, \lambda}$ will be done entirely analogously to Sec. V C.

Consider a point of $\mathcal{C}_{n, \lambda}$ represented by a collection of linear maps $X_{i}, Y_{j}, v_{i, \alpha}$, and $w_{i, \alpha}$, assuming the operator $X_{m-1} \ldots X_{1} X_{0} \in \operatorname{End}\left(V_{0}\right)$ has distinct non-zero eigenvalues $x_{1}^{m}, \ldots, x_{n}^{m}$. Such points form a dense subset $\mathcal{C}_{n, \lambda}^{\prime} \subset \mathcal{C}_{n, \lambda}$. As in the case $d=1$, there exist bases in each of $V_{i}$ diagonalizing the matrices $X_{i}$ as follows:

$$
X_{i}=\operatorname{diag}\left(x_{1}, \ldots, x_{n}\right) .
$$

Substituting this into relations (6.12), we obtain the following formulas:

$$
\begin{aligned}
\left(Y_{i}\right)_{j j}=p_{j}+\frac{1}{x_{j}} \sum_{r=0}^{i}\left(\lambda_{r}+\left(\psi_{j} \varphi_{j}\right)_{r r}\right)+\frac{1}{x_{j}} \sum_{r=0}^{m-1} \frac{r-m}{m}\left(\lambda_{r}+\left(\psi_{j} \varphi_{j}\right)_{r r}\right), \\
\left(Y_{i}\right)_{j k}=-\sum_{l=0}^{m-1} \frac{\left(\psi_{k} \varphi_{j}\right)_{i-l, i-l} x_{j}^{l} x_{k}^{m-l-1}}{x_{j}^{m}-x_{k}^{m}} \quad(j \neq k), \\
\left(v_{i, \alpha}\right)_{j}=\left(\varphi_{j}\right)_{\alpha i}, \quad\left(w_{i, \alpha}\right)_{j}=\left(\psi_{j}\right)_{i \alpha} \quad(\alpha=1, \ldots, d),
\end{aligned}
$$

where $i=0, \ldots, m-1$, and $\varphi_{j}$ and $\psi_{j}$ are the matrices of size $d \times m$ and $m \times d$, respectively, satisfying the constraint $\operatorname{tr}\left(\varphi_{j} \psi_{j}\right)=-|\lambda|(j=1, \ldots, n)$. As above, with each pair $\left(\varphi_{j}, \psi_{j}\right)$, we can associate a point $q_{j}$ of the symplectic variety 


$$
Q_{m, d}=\left\{(\varphi, \psi) \in \operatorname{Hom}\left(\mathbb{C}^{m}, \mathbb{C}^{d}\right) \oplus \operatorname{Hom}\left(\mathbb{C}^{d}, \mathbb{C}^{m}\right)|\operatorname{tr}(\varphi \psi)=-| \lambda \mid\right\} / \mathbb{C}^{\times},
$$

where the group $\mathbb{C}^{\times}$acts by $(\varphi, \psi) \mapsto\left(\kappa \varphi, \kappa^{-1} \psi\right)$. Thus we obtain a local parameterisation of $\mathcal{C}_{n, \lambda}^{\prime}$ by $\left\{x_{j}, p_{j}, q_{j}\right\}$.

Again, in the case $j \neq k$, we can rewrite the formula as

$$
\left(Y_{i}\right)_{j k}=\sum_{r \in I} \frac{\mu^{-i r} \operatorname{tr}\left(\varphi_{j} E^{r} \psi_{k}\right)}{m\left(x_{k}-\mu^{r} x_{j}\right)}, \quad E^{r}=\operatorname{diag}\left(1, \mu^{r}, \mu^{2 r}, \ldots, \mu^{(m-1) r}\right) .
$$

The symplectic structure (6.16) induces a symplectic structure on $\mathcal{C}_{n, \lambda}$, which we denote by $\omega_{\mathcal{C}_{n, \lambda}}$.

Proposition 6.6. The coordinates $x_{j}, p_{j}, q_{j}=\left(\varphi_{j}, \psi_{j}\right)$ are local Darboux coordinates on $\mathcal{C}_{n, \lambda}^{\prime}$, that is, the symplectic form $\omega_{\mathcal{C}_{n, \lambda}}$ can be written as

$$
\omega_{\mathcal{C}_{n, \lambda}}=\sum_{j=1}^{n}\left(m \mathrm{~d} p_{j} \wedge \mathrm{d} x_{j}+\operatorname{tr}\left(\mathrm{d} \psi_{j} \wedge \mathrm{d} \varphi_{j}\right)\right)
$$

where $\operatorname{tr}\left(\mathrm{d} \psi_{j} \wedge \mathrm{d} \varphi_{j}\right)=\sum_{r=0}^{m-1} \sum_{\beta=1}^{d} \mathrm{~d}\left(\psi_{j}\right)_{r \beta} \wedge \mathrm{d}\left(\varphi_{j}\right)_{\beta r}$ is understood as the corresponding form on $Q_{m, d}$.

The derivation of (6.28) is completely analogous to the proof of Proposition 5.8.

Let us consider the variety $\mathfrak{h}_{\mathrm{reg}} \times \mathfrak{h}^{*} \times\left(Q_{m, d}\right)^{n}$ equipped with the symplectic form (6.28). Substituting (6.24)-(6.26) into the Hamiltonians $H_{k, \beta}$, we get Poisson commuting functions on this symplectic variety. As in the case $d=1$, we obtain an integrable system in this way.

Proposition 6.7. Let $H_{k, \beta}=H_{k, \beta}(x, p, q)$ be the functions of the variables $(x, p, q) \in \mathfrak{h}_{\text {reg }} \times \mathfrak{h}^{*}$ $\times\left(Q_{m, d}\right)^{n}$, obtained by substituting formulas (6.24)-(6.26) into $H_{k, \beta}=-\sum_{i \in I} \operatorname{tr}\left(E_{\beta} w_{i} Y^{k} v_{i+k}\right)$. Then $H_{k, \beta}$ with $k=1, \ldots$, mn and $\beta=1, \ldots, d$ are functionally independent, Poisson commuting functions and hence define a completely integrable system. The Hamiltonian flows defined by $H_{k, \beta}$ extend from $\mathcal{C}_{n, \lambda}^{\prime}=\mathfrak{h}_{\text {reg }} \times \mathfrak{h}^{*} \times\left(Q_{m, d}\right)^{n} /\left(\mathbb{Z}_{m} S_{n}\right)$ to produce complete flows on the Calogero-Moser space $\mathcal{C}_{n, \lambda}=\mathcal{C}_{n \delta, \lambda}$ for quiver (3.12).

Proof. The number of integrals is $m n d$. It is the half of the dimension of the phase space $\mathfrak{h}_{\text {reg }}$ $\times \mathfrak{h}^{*} \times\left(Q_{m, d}\right)^{n}$. Thus it suffices to show their independence.

Note that there is an isomorphism $\mathcal{C}_{n, \lambda} \rightarrow \mathcal{C}_{n, \lambda^{\prime}}$, where $\lambda^{\prime}=\left(-\lambda_{m-1}, \ldots,-\lambda_{0}\right)$, defined by

$$
X_{i} \rightarrow Y_{-i-1}, \quad Y_{i} \rightarrow X_{-i-1}, \quad v_{i, \alpha} \rightarrow v_{-i, \alpha}, \quad w_{i, \alpha} \rightarrow-w_{-i, \alpha} .
$$

Under this isomorphism, the Hamiltonians $H_{k, \beta}$ take the form

$$
\widetilde{H}_{k, \beta}=\sum_{i \in I} w_{i+k, \beta} X^{k} v_{i, \beta}=\sum_{i \in I} \sum_{j=1}^{n}\left(\varphi_{j}\right)_{\beta, i}\left(\psi_{j}\right)_{i+k, \beta} x_{j}^{k} .
$$

Therefore, it is enough to proof the independence of the functions $\widetilde{H}_{k, \beta}$ in the neighbourhood of a point of $\mathfrak{h}_{\text {reg }} \times \mathfrak{h}^{*} \times\left(Q_{m, d}\right)^{n}$.

Consider $\widetilde{H}_{k}:=\sum_{\beta=1}^{d} \widetilde{H}_{k, \beta}$ for $k=m p, p=1, \ldots, n$,

$$
\widetilde{H}_{m p}=\sum_{j=1}^{n} \operatorname{tr}\left(\varphi_{j} \psi_{j}\right) x_{j}^{m p}=-|\lambda| \sum_{j=1}^{n} x_{j}^{m p} .
$$

Since $x_{i}^{m} \neq x_{j}^{m} \neq 0$ for $i \neq j$ on $\mathfrak{h}_{\text {reg }}$, the variables $x_{1}, \ldots, x_{n}$ can be expressed locally via $\widetilde{H}_{m p}$. Now let $k=m p-q$, where $p=1, \ldots, n$ and $q=0, \ldots, m-1$. Then

$$
\widetilde{H}_{m p-q, \beta}=\sum_{j=1}^{n} z_{j, \beta, q} x_{j}^{m p-q}, \quad \text { where } \quad z_{j, \beta, q}=\sum_{i \in I}\left(\varphi_{j}\right)_{\beta, i}\left(\psi_{j}\right)_{i-q, \beta} .
$$


Fixing $\beta$ and $q$, we obtain a system of linear equations with a Vandermonde matrix. It follows that locally $z_{j, \beta, q}$ are functions of $\widetilde{H}_{k, \beta}, k \leqslant m n$. Thus it suffices to show that there are $m n d$ independent functions on $\mathfrak{h}_{\text {reg }} \times \mathfrak{h}^{*} \times\left(Q_{m, d}\right)^{n}$ among $x_{j}$ and $z_{j, \beta, q}, j=1, \ldots, n, \beta=1, \ldots, d$, and $q=0, \ldots, m-1$.

For any fixed $j$, the functions $z_{j, \beta, 0}$ satisfy the constraint $\sum_{\beta=1}^{d} z_{j, \beta, 0}=-|\lambda|$. Clearly, we only need to prove that among $z_{j, \beta, q}$ with $\beta=1, \ldots, d, q=0, \ldots, m-1$ there exist $m d-1$ functions which are independent as functions on $Q_{m, d}$.

Let us first consider $z_{j, \beta, q}$ as functions on a lager space $\widehat{Q}_{m, d}=\operatorname{Hom}\left(\mathbb{C}^{m}, \mathbb{C}^{d}\right) \oplus \operatorname{Hom}\left(\mathbb{C}^{d}, \mathbb{C}^{m}\right)$. Choose the point $P \in \widehat{Q}_{m, d}$ with the following coordinates:

$$
\left(\varphi_{j}\right)_{\beta, i}=-|\lambda| / d, \quad\left(\psi_{j}\right)_{i, \beta}=\delta_{i, 0} .
$$

Since $\frac{\partial z_{j, \beta, q}}{\partial\left(\varphi_{j}\right)_{\alpha, r}}=\delta_{\alpha \beta} \delta_{r q}$ at the point $P$, the differentials $d z_{j, \beta, q}(\beta=1, \ldots, d$ and $q=0, \ldots, m-1)$ are linearly independent elements of $T_{P}^{*} \widehat{Q}_{m, d}$. Note that $P$ belongs to the hypersurface $H \subset \widehat{Q}_{m, d}$ defined by the equation $\sum_{\beta=1}^{d} z_{j, \beta, 0}=-|\lambda|$. Therefore, when restricted to $T_{P} H$, these differentials will span a subspace in $T_{P}^{*} H$ of dimension ( $m d-1$ ). It is clear that the same will be true in a neighbourhood of $P$ in $H$. This means that among $z_{j, \beta, q}$ there are $m d-1$ elements which are independent as functions on $H$. It follows that they are also independent as functions on $Q_{m, d}=H / \mathbb{C}^{\times}$.

It is convenient to consider the Hamiltonians

$$
H_{k}:=\sum_{\alpha=1}^{d} H_{k, \alpha}=-\sum_{i \in I} \operatorname{tr}\left(w_{i} Y^{k} v_{i+k}\right) .
$$

They are polynomials of the order $k$ in the momentum variables $p_{i}$. For $k=m p$, their leading terms are $H_{m p}=|\lambda| \sum_{j=1}^{n} p_{j}^{m p}$. Note that $H_{m p}$ are functions of $p_{j}, x_{j}$, and $\left(\psi_{k} \varphi_{j}\right)_{i i}$, where $i \in I$ and $j, k=1, \ldots, n$.

Example 6.8. In the case $m=1$, the first two Hamiltonians have the form

$$
H_{1}=|\lambda| \sum_{i=1}^{n} p_{i}, \quad H_{2}=|\lambda| \sum_{i=1}^{n} p_{i}^{2}+|\lambda| \sum_{i \neq j} \frac{\left(\psi_{i} \varphi_{j}\right)\left(\psi_{j} \varphi_{i}\right)}{\left(x_{i}-x_{j}\right)^{2}} .
$$

This is the spin Calogero-Moser system from Ref. 32. Its relation with the matrix KP hierarchy and the spaces $\mathcal{C}_{n, \lambda}$ has been considered in Refs. 38, 52, 6, and 13. Note that even in the $m=1$ case, our Theorem 6.5 gives more than, Ref. 6, Theorem 3.5 since we consider the full multicomponent KP hierarchy, while in Ref. 6, only the flows corresponding to $\partial_{t_{k}}=\sum_{\beta=1}^{d} \partial_{t_{k, \beta}}$ are considered.

\section{Spherical case}

For the multicomponent hierarchy (6.4) and (6.5), we can consider a sub-hierarchy consisting of the equations

$$
\frac{\partial L}{\partial t_{m j, \beta}}=\left[\left(L^{m j} R_{\beta}\right)_{+}, L\right], \quad \frac{\partial R_{\alpha}}{\partial t_{m j, \beta}}=\left[\left(L^{m j} R_{\beta}\right)_{+}, R_{\alpha}\right]
$$

$\left(j \in \mathbb{N}_{0}, \beta=1, \ldots, d\right)$ where the elements $L$ and $R_{\alpha}$ are assumed to have the following symmetry:

$$
\epsilon_{i} L=L \epsilon_{i-1}, \quad \epsilon_{i} R_{\alpha}=R_{\alpha} \epsilon_{i} .
$$

These are analogous to the conditions (2.23), and we will call this the multicomponent spherical sub-hierarchy.

Once again, solutions of this sub-hierarchy will be constructed by using representations of quivers.

In Sec. VI B, we constructed the Calogero-Moser space $\mathcal{C}_{\alpha, \lambda}$ associated with quiver 3.12. Let us now consider quiver (3.13) and write $\mathcal{C}_{\alpha, \lambda}^{s p h}$ for the corresponding Calogero-Moser space. For simplicity, we restrict to the case $\ell=0$, so we have $d$ arrows $b_{0,1}, \ldots, b_{0, d}$, all going from 0 to $\infty$. 
In this case, the variety $\mathcal{C}_{\alpha, \lambda}^{s p h}$ can be viewed as a symplectic subvariety of $\mathcal{C}_{\alpha, \lambda}$ by setting $v_{i}=w_{i}=0$ $(i \neq 0)$ in formulas (6.12)-(6.14). By Proposition 3.1

$$
\operatorname{dim} \mathcal{C}_{\alpha, \lambda}^{s p h}=2 d \alpha_{0}-\sum_{i \in I}\left(\alpha_{i}-\alpha_{i+1}\right)^{2}
$$

Now for $\left(X, Y, v_{0}, w_{0}\right) \in \mathcal{C}_{\alpha, \lambda}^{s p h}$, consider the element

$$
M=1-\epsilon_{0} w_{0}(X-x)^{-1}(Y-y)^{-1} v_{0} \epsilon_{0} .
$$

Since it commutes with each of $\epsilon_{i}$, the operators

$$
L=M y M^{-1}, \quad R_{\alpha}=M E_{\alpha} M^{-1}
$$

will have symmetry (6.31).

Theorem 6.9. Let $X, Y, w_{0}$, and $v_{0}$ satisfy Eqs. (6.18) and (6.19) with $k=m j$ (where $v_{i}=w_{i}=0$ for $i \neq 0)$. Then the elements $L, R_{\alpha}$ given by (6.32) and (6.33) satisfy the equations of the multicomponent spherical sub-hierarchy (6.30). Therefore solutions of the hierarchy can be found by integrating flows (6.18) and (6.19) with an arbitrary initial point in $\mathcal{C}_{\alpha, \lambda}^{s p h}$.

Proof. Flows (6.18) and (6.19) preserve the subspace $\mathcal{C}_{\alpha, \lambda}^{s p h} \subset \mathcal{C}_{\alpha, \lambda}$ defined by the conditions $v_{i}$ $=0, w_{i}=0(i \neq 0)$. Therefore the proof reduces to the application of Proposition 6.4 in the case $k=m j$.

The restriction of flows (6.18) and (6.19) with $k=m j$ onto $\mathcal{C}_{\alpha, \lambda}^{s p h}$ is given by the Hamiltonians

$$
h_{j, \beta}=\left.H_{m j, \beta}\right|_{\mathcal{C}_{\alpha, \lambda}^{s p h}}=-w_{0, \beta} Y^{m j} v_{0, \beta} \quad\left(j \in \mathbb{N}_{0}, \beta=1, \ldots, d\right) .
$$

Consider the principal Hamiltonians $h_{j}:=\sum_{\beta=1}^{d} h_{j, \beta}$. Note that $h_{j}=\frac{|\lambda|}{m} \operatorname{tr} Y^{m j}=|\lambda| \operatorname{tr} \widehat{Y}^{j}$, where $\widehat{Y}=Y_{0} Y_{1} \cdots Y_{m-1}$.

Let us now consider the special case $\alpha_{0}=\alpha_{1}=\cdots=\alpha_{m-1}=n$, writing $\mathcal{C}_{n, \lambda}^{s p h}$ for $\mathcal{C}_{n \delta, \lambda}^{s p h}$. This is a 2 nd-dimensional symplectic variety with coordinates $\left\{x_{j}, p_{j}, q_{j}\right\}$, where $q_{j}=\left(\varphi_{j}, \psi_{j}\right) \in Q_{1, d}$ with

$$
Q_{1, d}=\left\{(\varphi, \psi) \in\left(\mathbb{C}^{d}\right) \oplus\left(\mathbb{C}^{d}\right)^{*}|\operatorname{tr}(\varphi \psi)=\psi \varphi=-| \lambda \mid\right\} / \mathbb{C}^{\times}, \quad \operatorname{dim} Q_{1, d}=2 d-2 .
$$

A generic point of $\mathcal{C}_{n, \lambda}^{s p h}$ in these coordinates is described by the formulas

$$
\begin{aligned}
& \left(X_{i}\right)_{j k}=x_{j} \delta_{j k}, \\
& \left(Y_{i}\right)_{j j}=p_{j}+\frac{1}{x_{j}}\left(\sum_{r=0}^{i} \lambda_{r}-\sum_{r=0}^{m-1} \frac{m-r}{m} \lambda_{r}\right), \\
& \left(Y_{i}\right)_{j k}=-\frac{x_{j}^{i} x_{k}^{m-i-1}}{x_{j}^{m}-x_{k}^{m}} \psi_{k} \varphi_{j}, \\
& \left(v_{0 \alpha}\right)_{j}=\left(\varphi_{j}\right)_{\alpha}, \quad\left(w_{0 \alpha}\right)_{j}=\left(\psi_{j}\right)_{\alpha},
\end{aligned}
$$

where $i=0, \ldots, m-1$. A calculation similar to the proof of Proposition 5.8 shows that the symplectic form on $\mathcal{C}_{\alpha, \lambda}^{s p h}$ looks as $\omega_{s p h}=\sum_{j=1}^{n}\left(m \mathrm{~d} p_{j} \wedge \mathrm{d} x_{j}+\mathrm{d} \psi_{j} \wedge \mathrm{d} \varphi_{j}\right)$. By substituting formulas (6.34)-(6.37) into the above $h_{j, \beta}$, we obtain Poisson commuting functions $h_{j, \beta}(x, p, q)$ on $\mathfrak{h}_{\text {reg }} \times \mathfrak{h}^{*} \times\left(Q_{1, d}\right)^{n}$. By repeating the proof of Proposition 6.7 with $q=0$, one proves that $h_{j, \beta}$ with $j=1, \ldots, n$ and $\beta=1, \ldots, d$ are functionally independent. Thereby we obtain the analogous result.

Proposition 6.10. The functions $h_{j, \beta}(x, p, q)$ define a completely integrable system on $\mathfrak{h}_{\text {reg }} \times \mathfrak{h}^{*}$ $\times\left(Q_{1, d}\right)^{n}$. The Hamiltonian flows defined by $h_{j, \beta}$ extend from $\mathfrak{h}_{\mathrm{reg}} \times \mathfrak{h}^{*} \times\left(Q_{1, d}\right)^{n} /\left(\mathbb{Z}_{m} 2 S_{n}\right)$ to produce complete flows on $\mathcal{C}_{n, \lambda}^{s p h}$. 
Example 6.11. Consider the case $m=2$. The Hamiltonians $h_{p, \beta}$ describe a systems of $n$ particles with coordinates $x_{j}$ momenta $p_{j}$ and spin variables $\varphi_{j}=\left(\varphi_{j 1}, \ldots, \varphi_{j d}\right)^{\top}$ and $\psi_{j}=\left(\psi_{j 1}, \ldots, \psi_{j d}\right)$ satisfying $\psi_{j} \phi_{j}=-|\lambda|$. The first principal Hamiltonian of the system has the form

$$
h_{1}=|\lambda| \sum_{i=1}^{n} p_{i}^{2}-\frac{|\lambda| \lambda_{1}^{2}}{4} \sum_{i=1}^{n} \frac{1}{x_{i}^{2}}-\frac{|\lambda|}{2} \sum_{i<j}\left(\frac{1}{\left(x_{i}-x_{j}\right)^{2}}+\frac{1}{\left(x_{i}+x_{j}\right)^{2}}\right)\left(\psi_{i} \varphi_{j}\right)\left(\psi_{j} \varphi_{i}\right) .
$$

This is a $B_{n}$-variant of the Gibbons-Hermsen Hamiltonian (6.29), cf. Ref. 39.

\section{FURTHER DIRECTIONS AND LINKS}

Our results raise a number of questions; below we mention some of them and indicate further connections.

1. There are many aspects of the integrability for the usual KP hierarchy: $\tau$-function formalism, $W$-symmetries, (bi-)Hamiltonian approach, free-field realisation, and so on. Therefore it is a natural problem to find analogous notions for our hierarchy and to interpret the constructed solutions from that point of view.

2. The Calogero-Moser system for $G=\mathbb{Z}_{m} \backslash S_{n}$ admits trigonometric and elliptic versions for $m=2$ and $m=2,3,4,6$, respectively, see Ref. 30. In such cases, we expect these systems to be related to certain generalisations of the KP hierarchy. There is also a natural $q$-analogue related to multiplicative preprojective algebras of Crawley-Boevey and Shaw. ${ }^{21}$ These questions will be a subject of future work.

3. There is a link between the Calogero-Moser spaces for the cyclic quiver and the families of bispectral differential operators of Bessel type from Refs. 24 and 3. The key to that link is the fact that $\epsilon_{\ell} y^{m} \epsilon_{\ell}$ can be interpreted as a generalised Bessel operator (the classical Bessel operator corresponds to $m=2$ ). Then one can use the operator (4.36) as a dressing and construct bispectral differential operators in the form $\epsilon_{\ell} M p\left(y^{m}\right) M^{-1} \epsilon_{\ell}$ for suitable polynomials $p$. The details will appear elsewhere.

Note that Rothstein suggested in Ref. 46 a description of bispectral operators of Bessel type in terms of Wilson's Calogero-Moser spaces $\mathcal{C}_{n}$. However, it can be shown that the formulas in Ref. 46 correspond only to an open dense subset $\mathcal{C}_{n, \lambda}^{\prime} \subset \mathcal{C}_{n, \lambda}$. Also, the link with the Calogero-Moser problem for $\mathbb{Z}_{m} \imath S_{n}$ was not observed there.

4. Our results can be used to prove a conjecture of Etingof and Rains. Namely, in Ref. 31, the authors study algebraically integrable operators with $\mathbb{Z}_{m}$-symmetry. For the case of rational coefficients, they conjecture that the poles of such operators should be equilibrium points of the Calogero-Moser system for $\mathbb{Z}_{m}>S_{n}$ (see Ref. 31, Conjecture 4.10). This is related to our results as follows. Let $\mathcal{C}_{n, \lambda}$ be the Calogero-Moser space for quiver (3.15) with $\ell=0$ and let $\left(X, Y, v_{0}, w_{0}\right) \in \mathcal{C}_{n, \lambda}$ be an equilibrium point for the Hamiltonian $H_{m}$. Then we have $\partial_{t_{m}} M=0$, where $M$ is (4.36), implying $\left(L^{m}\right)_{-}=0$. Thus $D=\epsilon_{0} L^{m} \epsilon_{0}$ gives a purely differential operator. One has to restrict to the case $k_{i} \in \mathbb{Z} \tau$ to ensure that the operator $D$ is algebraically integrable. Let us assume that the matrix $X$ has distinct non-zero eigenvalues $\left\{\mu^{r} x_{j}\right\}, j=1, \ldots, n, r=0, \ldots, m-1$, and so $\left(x_{1}, \ldots, x_{n}\right) \in \mathbb{C}^{n}$ is an equilibrium point of the Calogero-Moser problem for $\mathbb{Z}_{m} \prec S_{n}$ by our assumption about $\left(X, Y, v_{0}, w_{0}\right)$. One can check that the resulting operator $D$ satisfies all the assumptions of (Ref. 31, Conjecture 4.10). This way we construct an algebraically integrable operator with $\mathbb{Z}_{m}$-symmetry starting from any equilibrium point of the Calogero-Moser problem. The full proof of the conjecture requires showing that the above construction produces all such operators. We will return to this problem elsewhere.

5. In the case, $m=1$ the Calogero-Moser spaces $\mathcal{C}_{n}$ are known to be related to the Bethe algebra of the quantum Gaudin model, see Ref. 42. It would therefore be interesting to relate the CalogeroMoser spaces $\mathcal{C}_{n, \lambda}$ with some generalisation of the Gaudin model. Note that a cyclotomic version of the Gaudin model has been proposed recently in Ref. 50.

6. Finally, it would be interesting to understand which of our results can be generalised to other quivers (see in particular Remark 4.8 above). 


\section{ACKNOWLEDGMENTS}

We are grateful to Yu. Berest, W. Crawley-Boevey, P. Etingof, and P. Iliev for stimulating discussions, and to G. Bellamy for useful comments. Both authors gratefully acknowledge the support by EPSRC under Grant No. EP/K004999/1.

${ }^{1}$ Airault, H., McKean, P. H., and Moser, J., "Rational and elliptic solutions of the Korteweg-de Vries equation and a related many-body problem," Commun. Pure Appl. Math. 30(1), 95-148 (1977).

2 Asensio, J. M., Van den Bergh, M., and Van Ostaeyen, F., "A new algebraic approach to microlocalization of filtered rings," Trans. Am. Math. Soc. 316(2), 537-553 (1989).

${ }^{3}$ Bakalov, B., Horozov, E., and Yakimov, M., "Bispectral algebras of commuting ordinary differential operators," Commun. Math. Phys. 190(2), 331-373 (1997); e-print arXiv:q-alg/9602011.

${ }^{4}$ Baranovsky, V., Ginzburg, V., and Kuznetsov, A., "Quiver varieties and a noncommutative $\mathbb{P}^{2}$," Compos. Math. 134(3), 283-318 (2002); e-print arXiv:math/0103068 [math.AG].

${ }^{5}$ Baranovsky, V., Ginzburg, V., and Kuznetsov, A., "Wilson's Grassmannian and a noncommutative quadric," Int. Math. Res. Not. 21, 1155-1197 (2003); e-print arXiv:math/0203116 [math.AG].

${ }^{6}$ Bergvelt, M., Gekhtman, M., and Kasman, A., "Spin Calogero particles and bispectral solutions of the matrix KP hierarchy," Math. Phys. Anal. Geom. 12(2), 181-200 (2009); e-print arXiv:math/0102190 [math.QA].

${ }^{7}$ Berest, Yu. and Wilson, G., "Automorphisms and ideals of the Weyl algebra," Math. Ann. 318(1), 127-147 (2000); e-print arXiv:math/0102190 [math.QA].

${ }^{8}$ Berest, Yu. and Wilson, G., "Ideal classes of the Weyl algebra and noncommutative projective geometry (with an appendix by Michel Van den Bergh)," Int. Math. Res. Not. 26, 1347-1396 (2002); e-print arXiv:math/0104248 [math.AG].

${ }^{9}$ Bordner, J. A., Corrigan, E., and Sasaki, R., "Calogero-Moser models. I: A new formulation," Prog. Theor. Phys. 100(6), 1107-1129 (1998); e-print arXiv:hep-th/9805106.

${ }^{10}$ Bordner, J. A., Sasaki, R., and Takasaki, K., "Calogero-Moser models. II: Symmetries and foldings,” Prog. Theor. Phys. 101(3), 487-518 (1999).

${ }^{11}$ Berest, Yu. and Chalykh, O., "A $A_{\infty}$-modules and Calogero-Moser spaces," J. Reine Angew. Math. 607, 69-112 (2007); e-print arXiv:math/0410194 [math.QA].

${ }^{12}$ Berest, Yu., Chalykh, O., and Eshmatov, F., "Recollement of deformed preprojective algebras and the Calogero-Moser correspondence,” Moscow Math. J. 8(1), 21-37 (2008); e-print arXiv:0706.3006 [math.RT].

${ }^{13}$ Bielawski, R. and Pidstrygach, V., "On the symplectic structure of instanton moduli spaces," Adv. Math. 226(3), 2796-2824 (2011); e-print arXiv:0812.4918 [math.SG].

${ }^{14}$ Bocklandt, R. and Le Bruyn, L., "Necklace Lie algebras and noncommutative symplectic geometry," Math. Z. 240(1), 141-167 (2002); e-print arXiv:math/0010030 [math.AG].

${ }^{15}$ Chudnovsky, V. D. and Chudnovsky, V. G., "Pole expansions of nonlinear partial differential equations," Il Nuovo Cimento B 40(2), 339-353 (1977).

${ }^{16}$ Crawley-Boevey, W. and Holland, M., "Noncommutative deformations of Kleinian singularities," Duke Math. J. 92(3), 605-635 (1998).

${ }^{17}$ Crawley-Boevey, W., Representations of quivers, preprojective algebras and deformations of quotient singularities, lectures at the workshop on quantizations of Kleinian singularities, Oberwolfach, May 1999, available at: http://www1.maths.leeds.ac.uk/pmtwc/dmvlecs.pdf.

${ }^{18}$ Crawley-Boevey, W., "Geometry of the moment map for representations of quivers," Compos. Math. 126(3), 257-293 (2001).

${ }^{19}$ Crawley-Boevey, W., "Decomposition of Marsden-Weinstein reductions for representations of quivers," Compos. Math. 130(2), 225-239 (2002); e-print arXiv:math/0007191 [math.AG].

${ }^{20}$ Crawley-Boevey, W., "Poisson structures on moduli spaces of representations," J. Algebra 325, 205-215 (2011).

${ }^{21}$ Crawley-Boevey, W. and Shaw, P., "Multiplicative preprojective algebras, middle convolution and the Deligne-Simpson problem,” Adv. Math. 201(1), 180-208 (2006); e-print arXiv:math/0404186 [math.RA].

22 Date, E., Jimbo, M., Kashiwara, M., and Miwa, T., "Transformation groups for soliton equations: IV. A new hierarchy of soliton equations of KP-type," Physica D 4(3), 343-365 (1982).

${ }^{23}$ Dickey, L., Soliton Equations and Hamiltonian Systems, Advanced Series of Mathematical Physics Vol. 26, 2nd ed. (World Scientific, 2003).

${ }^{24}$ Duistermaat, J. J. and Grünbaum, F. A., "Differential equations in the spectral parameter," Commun. Math. Phys. 103(2), 177-240 (1986).

${ }^{25}$ Dunkl, F. C. and Opdam, M. E., "Dunkl operators for complex reflection groups,” Proc. London Math. Soc. 86(1), 70-108 (2003); e-print arXiv:math/0108185 [math.RT].

${ }^{26}$ Eshmatov, F., "DG-models of projective modules and Nakajima quiver varieties," Homol., Homotopy Appl. 9(2), 177-208 (2007); e-print arXiv:math/0604011 [math.QA].

${ }^{27}$ Etingof, P. and Ginzburg, V., "Symplectic reflection algebras, Calogero-Moser space, and deformed Harish-Chandra homomorphism," Invent. Math. 147(2), 243-348 (2002); e-print arXiv:math/0011114 [math.AG].

${ }^{28}$ Etingof, P., "Calogero-Moser systems and representation theory," in Zurich Lectures in Advanced Mathematics (EMS, 2007).

${ }^{29}$ Etingof, P. and Ma, X., "Lecture notes on Cherednik algebras," e-print arXiv:1001.0432 [math.RT] (2010).

${ }^{30}$ Etingof, P., Felder, G., Ma, X., and Veselov, A., "On elliptic Calogero-Moser systems for complex crystallographic reflection groups," J. Algebra 329, 107-129 (2011); e-print arXiv:1003.4689 [math.QA].

${ }^{31}$ Etingof, P. and Rains, E., "On algebraically integrable differential operators on an elliptic curve," Symmetry Integrability Geom. Methods Appl. 7, 062 (2011); e-print arXiv:1011.6410 [math-ph]. 
${ }^{32}$ Gibbons, J. and Hermsen, T., “A generalisation of the Calogero-Moser system,” Physica D 11, 337-348 (1984).

${ }^{33}$ Heckman, J. G., "A remark on the Dunkl differential-difference operators," in Harmonic Analysis on Reductive Groups, Progress in Mathematics Vol. 101 (Birkhäuser, Boston, 1991), pp. 181-191.

${ }^{34}$ Hodges, J. T., "Noncommutative deformations of type-A Kleinian singularities," J. Algebra 161, 271-290 (1993).

${ }^{35} \mathrm{Kac}, \mathrm{G}$. V., "Infinite root systems, representations of graphs and invariant theory," Invent. Math. 56, 57-92 (1980).

${ }^{36}$ Kapustin, A., Kuznetsov, A., and Orlov, D., "Noncommutative instantons and twistor transform," Commun. Math. Phys. 221(2), 385-432 (2001); e-print arXiv:hep-th/0002193.

${ }^{37}$ Krichever, M. I., "On rational solutions of the Kadomtsev-Petviashvili equation and integrable systems of N particles on the line," Funct. Anal. Appl. 12(1), 59-61 (1978) [Funkts. Analiz i ego Pril. 12, 76-78 (1978) (in Russian)].

${ }^{38}$ Krichever, M. I., Babelon, O., Billey, E., and Talon, M., "Spin generalization of the Calogero-Moser system and the matrix KP equation," in Topics in Topology and Mathematical Physics, American Mathematical Society Translation Series 2 Vol. 170 (American Mathematical Society, 1995), pp. 83-119; e-print arXiv:hep-th/9411160.

${ }^{39} \mathrm{Li}$, C. L. and Xu, P., "A class of integrable spin Calogero-Moser systems," Commun. Math. Phys. 231(2), 257-286 (2002).

${ }^{40}$ Marsden, J. and Weinstein, A., "Reduction of symplectic manifolds with symmetry," Rep. Math. Phys. 5(1), 121-130 (1974).

${ }^{41}$ Martino, M., "Stratification of Marsden-Weinstein reductions for representations of quivers and deformations of symplectic quotient singularities," Math. Z. 258(1), 1-28 (2008); e-print arXiv:math/0603562 [math.RT].

${ }^{42}$ Mukhin, E., Tarasov, V., and Varchenko, A., "Bethe algebra of Gaudin model, Calogero-Moser space, and Cherednik algebra," Int. Math. Res. Not. 5, 1174-1204 (2014); e-print arXiv:0906.5185 [math.QA].

${ }^{43}$ Olive, D. and Turok, N., "The symmetries of Dynkin diagrams and the reduction of Toda field equations," Nucl. Phys. B 215(4), 470-494 (1983).

${ }^{44}$ Olshanetsky, A. M. and Perelomov, M. A., "Completely integrable Hamiltonian systems connected with semisimple Lie algebras," Invent. Math. 37(2), 93-108 (1976).

${ }^{45}$ Olshanetsky, A. M. and Perelomov, M. A., "Classical integrable finite-dimensional systems related to Lie algebras," Phys. Rep. 71(5), 313-400 (1981).

${ }^{46}$ Rothstein, M., "Explicit formulas for the Airy and Bessel bispectral involutions in terms of Calogero-Moser pairs," in The Bispectral Problem, CRM Proceedings and Lecture Notes Vol. 14 (American Mathematical Society, 1998), pp. 105-110; e-print arXiv:q-alg/9611027.

${ }^{47}$ Sato, M., "Soliton equations as dynamical systems on an infinite dimensional Grassmann manifold," RISM Kôkyûroku 439, 30-46 (1981).

${ }^{48}$ Shephard, C. G. and Todd, A. J., "Finite unitary reflection groups," Can. J. Math. 6, 274-304 (1954).

${ }^{49}$ M. Van den Bergh, "Double Poisson algebras," Trans. Am. Math. Soc. 360(11), 5711-5769 (2008); e-print arXiv:math/0410528 [math.QA].

${ }^{50}$ Vicedo, B. and Young, C. A. S., "Cyclotomic Gaudin models: Construction and Bethe ansatz," Commun. Math. Phys. 343(3), 971-1024 (2016); e-print arXiv:1409.6937 [math.QA].

${ }^{51}$ Wilson, G., "Collisions of Calogero-Moser particles and an adelic Grassmannian (with an appendix by I. G. Macdonald)," Invent. Math. 133(1), 1-41 (1998).

${ }^{52}$ Wilson, G., "Notes on the vector adelic Grassmannian,” e-print arXiv:1507.00693 [math.SG] (2009). 